\title{
Graviton Signals in Central Production at the LHC
}

\author{
Rahul Basu and Tanumoy Mandal \\ The Institute of Mathematical Sciences, Chennai, Tamil Nadu 600113, India \\ Correspondence should be addressed to Tanumoy Mandal; tanumoy@imsc.res.in
}

Received 18 February 2013; Revised 1 May 2013; Accepted 13 May 2013

Academic Editor: P. Bussey

Copyright (C) 2013 R. Basu and T. Mandal. This is an open access article distributed under the Creative Commons Attribution License, which permits unrestricted use, distribution, and reproduction in any medium, provided the original work is properly cited.

\begin{abstract}
We study central production, in the inclusive case, to look for graviton signals in a large extra dimensional model using dilepton and diphoton channels. We carefully analyze signal and possible standard model background processes and study the feasibility of such new physics searches in a relatively clean environment as in central production where the proton fragments are mostly emitted in the forward direction, and there is a clear rapidity gap between them and the centrally produced system. Our analysis shows that the LHC with $14 \mathrm{TeV}$ center of mass energy and $100 \mathrm{fb}^{-1}\left(300 \mathrm{fb}^{-1}\right)$ of integrated luminosity can probe the effective gravity scale up to $3.6 \mathrm{TeV}(4.4 \mathrm{TeV})$ in both the dilepton and diphoton channels.
\end{abstract}

\section{Introduction}

The standard model (SM) has been extremely compatible with all the experimental tests so far. However, there are many theoretical attempts to go beyond the SM in order to answer some fundamental issues in the SM, namely, the hierarchy problems, number of fermion generations. Theories with extra spatial dimensions have been proposed to answer the hierarchy problems of the SM $[1,2]$. In what follows, in this paper, we look for the graviton signals as predicted in the large extra dimensional model (proposed by Arkani-Hamed et al. [1]) in the central diffractive processes at the LHC.

Forward physics has been used with great success in studying the (SM) physics at colliders. In particular, hadronelectron ring accelerator (HERA) has used forward detectors to greatly enhance our knowledge of perturbative quantum chromodynamics (QCD) and its effects in deep inelastic scattering (DIS) experiments. Moreover, the observation of diffractive events, those with large rapidity gaps between the final state particles, have been one of the most interesting aspects of HERA physics.

In the last decade, the importance of forward physics has been recognized for Large Hadron Collider (LHC) physics too. This has resulted in a proposal to install forward particle detectors at ATLAS and CMS in order to detect protons emitted in the forward direction, implying the existence of a centrally produced system with a rapidity gap between it and the protons $[3,4]$. More specifically, we are talking of a process of the kind $p+p \rightarrow p+M+p$, also known as Central Exclusive Production (CEP). In this case, the outgoing protons are emitted with barely any loss of energy (typically less than $2 \%$ of their longitudinal momenta) along with a centrally produced system $M$ with a rapidity gap between it and the outgoing protons on either side. This is a clean production of the central system since it corresponds to just two forward protons in the final state along with the decay products used to identify the central particle $M$ and no hadronic activity between them. This has therefore been described as being like a vacuum fluctuation as the two protons pass each other, producing the central particle. Some good review on the phenomenology of exclusive processes can be found in [5-7]. Recently, in [8] a general framework of exclusive double diffractive processes and its prospects are discussed.

One of the main aim of the forward physics at the LHC is to look for the exclusive Higgs production events. First in [9], it was pointed out that Higgs can be produced in diffractive processes with rapidity gaps on either side at hadron-hadron colliders. Later, this interesting scenario has been discussed in a series of papers [10-18] by several authors. Recently, to compute the background for Higgs searches, in [19], authors have estimated the exclusive $b \bar{b}$ pair production cross-section at the LHC. In exclusive processes, Higgs would be produced 
almost at rest through gluon-gluon fusion. The final state contains two protons and a Higgs and, since the process is exclusive, the invariant mass of the Higgs is directly related to the energy loss of the outgoing protons [20]. In exclusive production, measuring the energy loss of the protons, one can determine the mass of the central system without looking at its decay products.

It was discussed in [21], if the outgoing protons scatter through small angles, the two-gluon system is in a $J_{z}=0$, $\mathrm{C}$-even, $\mathrm{P}$-even state, where the $z$-axis is the proton beam axis. This means that any new resonance must carry the $0^{++}$ quantum number in CEP. In other words, this enables a clean determination of the quantum numbers of any new resonance [22].

With the possibility of tagging the forward outgoing protons, the LHC can be turned into an effective gluon-gluon, photon-proton, and photon-photon collider, giving rise to a major QCD and electroweak physics program [23, 24]. In addition, the possibility of producing quarkonium states like $\chi, J / \psi$, is something being envisaged at the ALICE detector. As discussed in $[25,26]$, the measurement of the two-body decay channels of $\chi_{c 0}$ to light mesons in an exclusive process can be used to study the dynamics of heavy quarkonia and to test the QCD framework of CEP.

However, all these studies require a QCD based model that allows us to couple the digluon system to the protons. The preferred model to describe that this is the socalled Durham model which we briefly describe in the next section. Before we do that however, we should point out that various other processes in the CEP scenario have recently been observed in the Tevatron at CDF which have given physicists the confidence that the Durham model is on the right track. Even though the Durham model was proposed almost a decade ago, it was not until 2006 that CDF saw diphoton production in the central rapidity region which was consistent with the gluon-gluon fusion Durham model [27]. Subsequently, CDF also found another exclusive process$e^{+} e^{-}$and $\mu^{+} \mu^{-}$production-produced by two photons [28]. Here, the Tevatron (and in future the LHC) acts as a photonphoton collider. These processes also allow the calibration of the forward proton detectors proposed by CMS and ATLAS. Quarkonium production of $J / \psi$ and $\psi(2 S)$ as well as $\chi_{c}^{0}$ have also been observed through their decay products [29]. Exclusive dijet production has been observed, at CDF [30] and D0 [31] in line with the predictions of the Durham model. These processes already seen at the Tevatron have given confidence that we can use digluon fusion in accordance with the Durham model to observe the signatures of new physics.

It was indicated in [32] that new physics like SUSY, extra dimensions, and so forth can be searched for in exclusive processes. In the exclusive configuration, various BSM signatures, namely, MSSM Higgs [33], charged Higgs [34], triplet Higgs [35], radions [36, 37], massive gravitons [37], long lived gluinos [38], effects of quantum gravity [39], and so forth, have been discussed in the literature. In this work, we study inclusive double diffractive process as a probe of large extra dimensions (the ADD model) using dilepton and diphoton channels via the exchange of KK graviton.
In Section 2, we briefly introduce the ADD model. In Section 3, we discuss diffractive processes and summarize the Durham model. In Section 4, we consider dilepton and diphoton production via KK graviton. We discuss important SM backgrounds in Section 5. In Section 6, we summarize our numerical results and present LHC discovery potential of extra dimensions. Finally, in Section 7, we offer our conclusions.

\section{The ADD Model}

In this section, we give a very brief introduction of the ADD model [1]. This model assumes that space is $4+n$ dimensional where $n$ is the number of extra spatial dimensions. All the $\mathrm{SM}$ particles are confined to the usual $(3+1) D$ spacetime which is called "brane," whereas gravity can propagate in the extra dimensions. In this model, the 4D Planck scale $\left(M_{\mathrm{Pl}} \sim 10^{19} \mathrm{GeV}\right)$ is a derived scale which is related to the fundamental Planck scale $\left(M_{S} \sim \mathrm{TeV}\right)$ by

$$
M_{\mathrm{Pl}}^{2}=V_{n} M_{S}^{2+n}, \quad V_{n}=(2 \pi R)^{n},
$$

where $V_{n}$ is the extra dimensional volume and $R$ is the compactification radius. An important consequence of the ADD model is the appearance of a tower of Kaluza-Klein (KK) modes as a solution to the linearized Einstein equation in $4+n$ dimensions. These KK modes are almost degenerate and separated in mass by $\mathcal{O}(1 / R)\left(1 / R \sim 10^{-4} \mathrm{eV}\right.$ to $\sim 100 \mathrm{MeV}$ for $n=2-7)$ terms. After KK decomposition, we have massive spin-2 KK gravitons $\left(h_{\mu \nu}^{k}\right)$ which have interactions with the brane localized SM states via the energy momentum tensor $T^{\mu \nu}$ of the SM

$$
\mathscr{L}=-\frac{\kappa}{2} \sum_{k} T^{\mu \nu}(x) h_{\mu \nu}^{k}(x),
$$

where $\kappa=\sqrt{16 \pi} / M_{\mathrm{Pl}}$ and the summation runs over all KK modes. The ADD model has been studied extensively both theoretically as well as experimentally. Recently, the extra dimensions are being searched for using dilepton and diphoton channels at the LHC, both by ATLAS $[40,41]$ and CMS [42, 43]. These searches have already put the lower bound on $M_{S}$ quite high.

\section{Diffractive Processes}

We consider two types of double diffractive (DD) mechanisms at the LHC as follows:

(1) central exclusive process (CEP): $p p \rightarrow p+M+p$ as shown in Figure 1(a);

(2) central inclusive process (CIP): $p p \rightarrow X+M+Y$ as shown in Figure 1(b).

Here, $M$ denotes the centrally produced system (we also denote the invariant mass of the central system with the same notation " $M$ ") and the "+" signs denote the presence of rapidity gaps between the system $M$ and the outgoing protons (in exclusive process) or proton dissociated products 


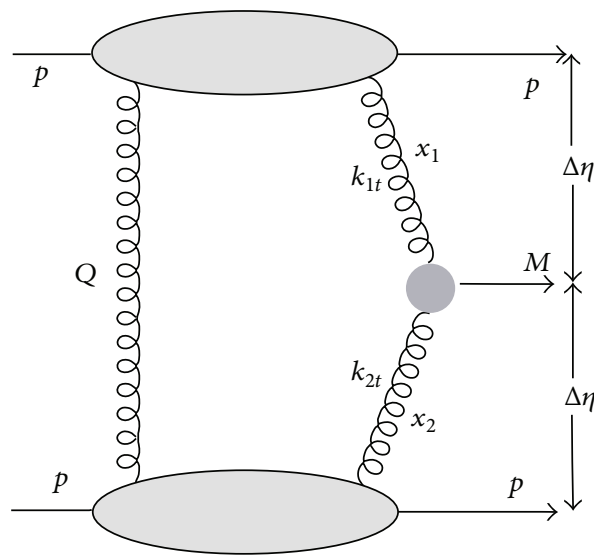

(a)

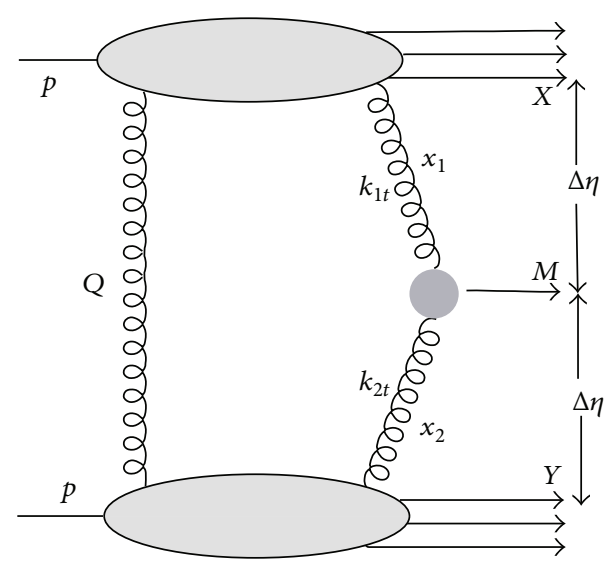

(b)

FIGURE 1: Double diffractive production of a system of mass $M$ with rapidity gap $\Delta \eta$ on either side via (a) the central exclusive process and (b) the central inclusive process at the LHC.

$X, Y$ (in inclusive process). The differential cross-sections for exclusive and inclusive DD processes shown in Figure 1 can be expressed in the folllowing factorized form [32]:

$$
M^{2} \frac{d^{2} \sigma}{d y d M^{2}}=\mathscr{L}_{g g}\left(M^{2}, y\right) \hat{\sigma}\left(M^{2}\right),
$$

where $\widehat{\sigma}$ is the cross-section of the hard subprocess which produces the color singlet system of invariant mass $M(g g \rightarrow$ $M)$ and $\mathscr{L}_{g g}$ is the effective luminosity for the production of a system of invariant mass $M$ at rapidity $y$. The total crosssection $\sigma(p p \rightarrow X+M+Y)$ can be calculated using

$$
\sigma=\int d y d M^{2} \frac{1}{M^{2}} \mathscr{L}_{g g}\left(M^{2}, y\right) \hat{\sigma}\left(M^{2}\right) .
$$

In our calculation, we keep $\mathscr{L}_{g g}$ and $\widehat{\sigma}$ in a factorized form as the effective luminosity is independent of the subprocess, and for different processes, we need to compute just the subprocess cross-sections. Although we keep $\mathscr{L}_{g g}$ and $\widehat{\sigma}$ in a factorized form, we must remember that there might exist specific selection rules for different configurations. In the exclusive configuration, the fusing $g g$ state obeys a special selection rule in the limit that the protons scatter through zero angle. The $z$-component of the angular momentum of a centrally produced system vanishes as the incoming state which consists of the fusing hard gluons has $J_{z}=0$ with positive $C$ and $P$ parity [32]. For inclusive production on the other hand, there is no such selection rule, and therefore, the production rate is larger than that for the exclusive production. Although the signals are very clear for CEP, the luminosity and the event rates are very small due to the restricted kinematics. A much larger phase space is available due to the dissociation of the incoming protons in the inclusive DD case. The luminosities for exclusive and inclusive processes can be computed using the known parton distribution functions (PDF) of the protons.

3.1. The Durham Model. To estimate $\mathscr{L}_{g g}$, we will use what is known as the "Durham model," described in [14, 15, 18, 32].
The $\mathscr{L}_{g g}$ depends on off-diagonal or skewed gluon distribution functions (off-diagonal, since we need to consider the coupling of two gluons with different momentum fractions, $x_{i}$ and $x_{i}^{\prime}$, with a proton). However, as we see, for $x_{i}^{\prime} \ll x_{i}$ and $k_{i t} \approx Q_{t}$, the relevant kinematic region for CEP, it is possible to approximate the off-diagonal distributions using the integrated gluon distribution function [44]. For CIP, the dominant (leading logarithm) contribution comes from the asymmetric configuration, $Q_{t}^{2} \ll k_{i t}^{2}$. At the partonic level, the inclusive production is equivalent to the exclusive production, and the unintegrated gluon distributions in partons may be calculated perturbatively in terms of nonforward BFKL amplitudes. The effective luminosity depends also on two survival probabilities of the rapidity gaps-one to the QCD radiation and another to the nonperturbative soft rescattering of the protons. The first is expressed as a Sudakov factor which ensures that the fusing gluons remain intact up to the hard scale $M / 2$. The second factor is included as an explicit multiplicative factor $\mathcal{S}^{2}$, known as the "gap survival factor." It accounts for the probability that apart from the perturbative processes, no other particle is produced via soft interactions between the protons [45-48]. In general, $\mathcal{S}^{2}$ is expected to depend on the kinematics of the process and can be calculated using various QCD based models [47-52]. Typically, for LHC energies, $\delta^{2}$ for CEP is estimated to be very small $[47,48]$ (e.g., in [32] authors found $\mathcal{S}^{2}=0.020(0.026)$ for $14 \mathrm{TeV}$ $(8 \mathrm{TeV})$ LHC). For CIP, relatively smaller absorption crosssection leads to a larger $\mathcal{S}^{2}$. For simplicity, in our analysis, we keep $\mathcal{S}^{2}=0.1$ for CIP, as a constant multiplicative factor.

We use the MSTW2008 LO parton density [53] in our computation. Since MSTW parametrization for the gluon distribution function $G(x, Q)=x g(x, Q)$ does not go below $Q=1 \mathrm{GeV}$, for $Q \leq 1 \mathrm{GeV}$, we take the following extrapolation function:

$$
G(x, Q)=\alpha(x) Q^{[2+\{\beta(x)-2\} Q]},
$$


so that for $Q^{2} \rightarrow 0, G(x, Q) \sim Q^{2}[18]$. Here, $\alpha$ and $\beta$ are the two functions of $x$ and can be computed using the following relations:

$$
\begin{gathered}
\beta(x)=\frac{1}{\left(1+\ln Q_{0}\right)}\left(\frac{G^{\prime}\left(x, Q_{0}\right)}{G\left(x, Q_{0}\right)}-\frac{2}{Q_{0}}\right)+2, \\
\alpha(x)=\frac{G\left(x, Q_{0}\right)}{Q_{0}^{\left[2+\{\beta(x)-2\} Q_{0}\right]}},
\end{gathered}
$$

where $G^{\prime}=\partial G / \partial Q$ and $G, G^{\prime}$ are evaluated at $Q_{0}=1 \mathrm{GeV}$. To compute the Sudakov factor, we use the LO $\alpha_{S}$ (strong coupling) with $\Lambda_{\mathrm{QCD}}=220 \mathrm{MeV}$ and $N_{f}=5$. The full formalism to calculate effective luminosity for exclusive and inclusive configuration is given in [32]. In Appendix A, we briefly present the computation of $\mathscr{L}_{g g}$ for CIP.

\section{Diffractive Production}

With diffractive processes being dominated by two-gluon exchange as shown in Figure 1, it is obvious that these processes would be observed only for the final states with a substantial coupling to a gluon pair. In this work, we consider the central production of KK gravitons $(G)$ via DD processes. The spin-2 gravitons can have three possible spin projections $J_{z}=0,1$, and 2 . Due to the presence of special $J_{z}=0$ selection rule in the exclusive configuration, graviton cannot be produced via leading order (LO) exclusive processes (although there is no $J_{z}=0$ point-like $g g$ coupling to a $2^{+}$graviton, it can still be produced at next to $\mathrm{LO}$ (NLO) in the exclusive configuration.) [32]. In the unitary gauge, the coupling of the spin- 0 projection of $G$ with two gluons is zero as the LO coupling of spin- 0 component with any two vector bosons is proportional to the square of the mass of the vector boson, and gluons are massless in this case [54]. By the Landau-Yang theorem $[55,56]$, it is impossible to produce a spin-1 particle via the fusion of two on-shell vector bosons, but the production is not forbidden if those vector bosons are off-shell. Though the gluons coming from protons are not purely on-shell, the degree of virtuality of the gluons is very small. Thus, the contribution in any process from spin-1 projection of graviton is subdominant [57]. Thus, the $J_{z}=2$ is the only possible spin projection that can couple to a gluon pair at LO. Therefore, in this paper, we discuss the LHC signatures of $G$ in inclusive configuration. In what follows, we analyze the diphoton and the dilepton channels via the exchange of $G$ arising in ADD type models [1] as a signal of extra dimension. We carefully consider these signal processes and the relevant background processes for them and look for regions of parameter space where the signal would dominate. Since these processes can only occur in inclusive configuration, the event rates could be substantial to search for at the LHC. Moreover, the nature of the intermediate particle and the coupling with SM particles reflect in the angular distribution of the final state particles.

4.1. Dilepton Production. We consider the process where dilepton pair is produced from gluon fusion via the exchange of $G$ as shown in Figure 2. The Feynman rules for this process

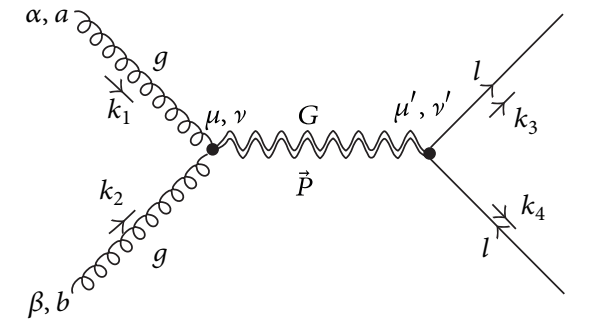

Figure 2: The subprocess Feynman diagram for dilepton production.

are taken from [54] where one can find definitions of all the terms which appear here. We also show these Feynman rules in Appendix B. The matrix element for this process is given by

$$
\begin{aligned}
i \mathscr{M}^{a b}= & \left(-\frac{i \kappa}{2} \delta^{a b}\right) \epsilon_{1}^{\alpha}\left(k_{1}\right) \epsilon_{2}^{\beta}\left(k_{2}\right) V_{\mu \gamma ; \alpha \beta} \\
& \times \frac{(i / 2) B^{\mu \nu ; \mu^{\prime} \nu^{\prime}}}{P^{2}-M_{G}^{2}+i \varepsilon}\left(-\frac{i \kappa}{8}\right) \bar{u}\left(k_{3}\right) \Gamma_{\mu^{\prime} \nu^{\prime}} \nu\left(k_{4}\right),
\end{aligned}
$$

where the coupling $\kappa$ is related to the Newton constant $G_{N}$ as $\kappa=\sqrt{16 \pi G_{N}}$. Since the reduced Planck mass $M_{\mathrm{Pl}}^{*}=$ $1 / \sqrt{8 \pi G_{N}}\left(M_{\mathrm{Pl}}^{*} \approx 2.4 \times 10^{18} \mathrm{GeV}\right)$, each KK mode coupling to the SM particles is Planck mass suppressed. A summation over high multiplicity of KK modes lying below the UV cutoff scale $M_{\mathrm{S}}$ compensates the suppression and gives rise to a substantial effective coupling strength. Therefore, we replace the graviton propagator in (7) by the following effective propagator:

$$
\mathscr{D}_{\text {eff }}(\widehat{s})=\sum_{k} \frac{i}{\left[\widehat{s}-\left(M_{G}^{2}\right)_{k}+i\left(\Gamma_{G}\right)_{k}\left(M_{G}\right)_{k}\right]},
$$

where $k$ sums over all $\mathrm{KK}$ towers below $M_{S}$ and $\Gamma_{G} M_{G}=\varepsilon$ and "hat" notation is used for subprocess quantities. Here, $\sqrt{\widehat{s}}=M_{l l}$ is the invariant mass of the lepton pair. Since, KK modes are quasicontinuous, this summation can be done by defining KK state density as shown in Appendix B [54]. For $n$ number of extra dimensions, considering the contributions from resonant and nonresonant KK states, the $\mathscr{D}_{\text {eff }}$ is given by

$$
\mathscr{D}_{\text {eff }}(\widehat{s})=\frac{16 \pi \widehat{s}^{n / 2-1}}{\kappa^{2} \Gamma(n / 2) M_{S}^{n+2}}\left[\pi+2 i I\left(\frac{M_{S}}{\sqrt{\widehat{s}}}\right)\right]
$$

where the real part comes from the summation over all resonant contributions below $M_{S}$ and the imaginary part is the summed contribution coming from all the nonresonant states. The definition of the $I$ function can be found in Appendix B. After summing over the spins of the final 
state leptons, the effective squared matrix element takes the following form:

$$
\begin{aligned}
|\mathscr{M}|^{2}= & \left(\frac{\kappa^{4}}{1024}\right)\left(\mathscr{D}_{\mathrm{eff}}\right)^{2}\left(\epsilon_{1}^{\alpha_{1}}\left(k_{1}\right) \epsilon_{1}^{* \alpha_{2}}\left(k_{1}\right)\right) \\
& \times\left(\epsilon_{2}^{\beta_{1}}\left(k_{2}\right) \epsilon_{2}^{* \beta_{2}}\left(k_{2}\right)\right) V_{\mu_{1} \nu_{1} ; \alpha_{1} \beta_{1}} V_{\mu_{2} \nu_{2} ; \alpha_{2} \beta_{2}} \\
& \times B^{\mu_{1} \nu_{1} ; \mu_{1}^{\prime} \nu_{1}^{\prime}} B^{\mu_{2} \nu_{2} ; \mu_{2}^{\prime} \nu_{2}^{\prime}} \\
& \times \operatorname{tr}\left[\left(\bar{u}\left(k_{3}\right) \Gamma_{\mu_{1}^{\prime} v_{1}^{\prime}} v\left(k_{4}\right)\right)\left(\bar{v}\left(k_{4}\right) \Gamma_{\mu_{1}^{\prime} \nu_{1}^{\prime}}^{\dagger} u\left(k_{3}\right)\right)\right] \delta_{a b} \delta^{a b} .
\end{aligned}
$$

In the zero mass limit of the produced lepton, the angular dependence of $|\mathscr{M}|^{2}$ takes a very simple form as follows:

$$
|\mathscr{M}|^{2}=\delta_{a b} \delta \delta^{a b} \frac{\kappa^{4}}{64}\left(\mathscr{D}_{\mathrm{eff}}\right)^{2} \widehat{s}^{4}\left(1-\cos ^{4} \theta\right),
$$

where $\theta$ is the scattering angle of the leptons in the center of mass (CM) frame of the G. Averaging $|\mathscr{M}|^{2}$ over eight colors and two polarizations of the initial gluons, one can evaluate the subprocess differential cross-section after including the phase space factor as

$$
\begin{aligned}
\frac{d \widehat{\sigma}\left(g g \rightarrow l^{+} l^{-}\right)}{d|\cos \theta|}= & \left(\frac{1}{32 \pi \widehat{s}}\right) \cdot\left(\frac{1}{8^{2}}\right) \cdot\left(\frac{1}{2^{2}}\right) \cdot 8 \cdot \frac{\kappa^{4}}{64}\left(\mathscr{D}_{\mathrm{eff}}\right)^{2} \\
& \times \widehat{s}^{4}\left(1-\cos ^{4} \theta\right) .
\end{aligned}
$$

If we consider that two types of leptons (i.e., electron and muon) are contributing in the dilepton final state, we should multiply the previous differential cross-section by an extra factor of two.

4.2. Diphoton Production. We consider the process where a photon pair is produced from gluon fusion via the exchange of $G$ as shown in Figure 3. As mentioned earlier, the Feynman rules for this process are taken from [54] where one can find definitions of all the terms which appear here and also can be found in Appendix B. The matrix element for this process is given by

$$
\begin{aligned}
i M^{a b}= & \left(-i \frac{\kappa}{2} \delta^{a b}\right) \epsilon_{1}^{\alpha}\left(k_{1}\right) \epsilon_{2}^{\beta}\left(k_{2}\right) V_{\mu \nu ; \alpha \beta} \frac{(i / 2) B^{\mu v ; \mu^{\prime} \nu^{\prime}}}{P^{2}-M_{G}^{2}+i \varepsilon} \\
& \times\left(-i \frac{\kappa}{2}\right) V_{\mu^{\prime} \nu^{\prime} ; \alpha^{\prime} \beta^{\prime}} \epsilon_{3}^{* \alpha^{\prime}}\left(k_{3}\right) \epsilon_{4}^{* \beta^{\prime}}\left(k_{4}\right) .
\end{aligned}
$$

After summing over all KK states up to $M_{S}$ contributing to a physical process and summing over two polarizations of the final state photons, the effective squared matrix element takes the following form:

$$
\begin{aligned}
|\mathscr{M}|^{2}= & \left(\frac{\kappa^{4}}{64}\right)\left(\mathscr{D}_{\mathrm{eff}}\right)^{2}\left(\epsilon_{1}^{\alpha_{1}}\left(k_{1}\right) \epsilon_{1}^{* \alpha_{2}}\left(k_{1}\right)\right)\left(\epsilon_{2}^{\beta_{1}}\left(k_{2}\right) \epsilon_{2}^{* \beta_{2}}\left(k_{2}\right)\right) \\
& \times\left(\epsilon_{3}^{* \alpha_{1}^{\prime}}\left(k_{3}\right) \epsilon_{3}^{\alpha_{2}^{\prime}}\left(k_{3}\right)\right)\left(\epsilon_{4}^{* \beta_{1}^{\prime}}\left(k_{4}\right) \epsilon_{4}^{\beta_{2}^{\prime}}\left(k_{4}\right)\right) \\
& \times V_{\mu_{1} \nu_{1} ; \alpha_{1} \beta_{1}} V_{\mu_{1}^{\prime} \nu_{1}^{\prime} ; \alpha_{1}^{\prime} \beta_{1}^{\prime}} V_{\mu_{2} \nu_{2} ; \alpha_{2} \beta_{2}} \\
& \times V_{\mu_{2}^{\prime} \nu_{2}^{\prime} ; \alpha_{2}^{\prime} \beta_{2}^{\prime}} B^{\mu_{1} \nu_{1} ; \mu_{1}^{\prime} \nu_{1}^{\prime}} B^{\mu_{2} \nu_{2} ; \mu_{2}^{\prime} \nu_{2}^{\prime}} \delta_{a b} \delta^{a b} .
\end{aligned}
$$

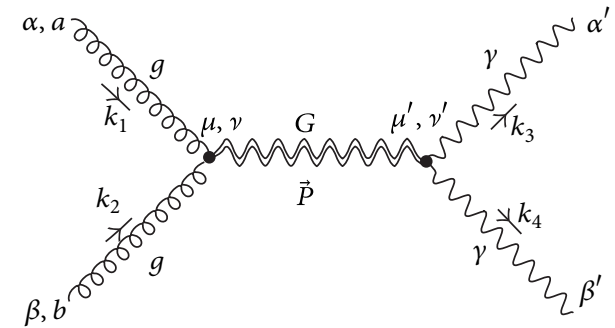

FIgUre 3: The subprocess Feynman diagram for diphoton production.

After doing some tedious algebra, the angular dependence of $|\mathscr{M}|^{2}$ takes the following form:

$$
|\mathscr{M}|^{2}=\delta_{a b} \delta \frac{\kappa^{4}}{64}\left(\mathscr{D}_{\mathrm{eff}}\right)^{2} \widehat{s}^{4}\left(1+6 \cos ^{2} \theta+\cos ^{4} \theta\right),
$$

where $\theta$ is the scattering angle of the photons in the CM frame of the $G$. Averaging $|\mathscr{M}|^{2}$ over eight colors and two polarizations of the initial gluons, one can evaluate the subprocess differential cross-section after including the phase space factor as

$$
\begin{aligned}
\frac{d \widehat{\sigma}(g g \rightarrow \gamma \gamma)}{d|\cos \theta|}= & \frac{1}{2} \cdot\left(\frac{1}{32 \pi \widehat{s}}\right) \cdot\left(\frac{1}{8^{2}}\right) \cdot\left(\frac{1}{2^{2}}\right) \cdot 8 \cdot \frac{\kappa^{4}}{64}\left(\mathscr{D}_{\mathrm{eff}}\right)^{2} \\
& \times \widehat{s}^{4}\left(1+6 \cos ^{2} \theta+\cos ^{4} \theta\right) .
\end{aligned}
$$

Here, we have included an extra $1 / 2$ factor because of the presence of two identical particles in the final state.

\section{SM Backgrounds}

In the diffractive configuration, there are possibilities of producing a central system from $g g, \gamma \gamma, q q$, or $W W$ fusion processes. In a same kinematic region, the effective luminosity for the $g g$ fusion $\left(\mathscr{L}_{g g}\right)$ is the largest at the LHC among all the alternatives, whereas the effective luminosities for $\gamma \gamma, q q$, or $W W$ fusion processes are much smaller compared to $\mathscr{L}_{q g}$. Estimations of effective luminosities for the $g g, \gamma \gamma$, and $W W$ can be found in [32] and for the qq in [58]. While computing the SM backgrounds for the dilepton and the diphoton signal channels, we can sometimes neglect $\gamma \gamma, q q$, and $W W$ initiated background processes due to the small luminosities of the fusing particles.

5.1. Dilepton Backgrounds. In Figure 4, we show the Feynman diagrams of some important background subprocesses for the dilepton channel proceed through $g g, \gamma \gamma, q q$, and $W W$ initial states. When one considers lepton pair production within the SM in the diffractive configuration, one would not expect a significant rate for a lepton pair unaccompanied by other particles (like radiation jets/photons or other SM particles). Although there is always a chance to miss those extra particles other than the lepton pair or to misidentify other particles as leptons, in our background computation, we do not include 


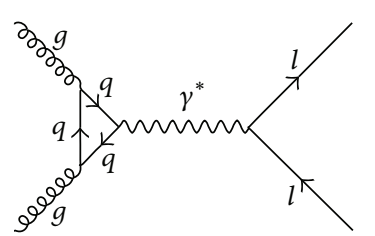

(a)

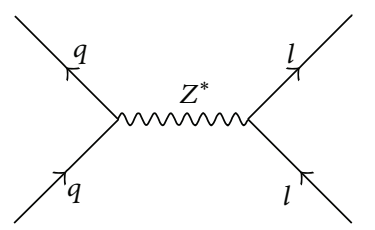

(e)

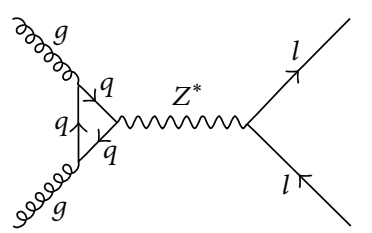

(b)

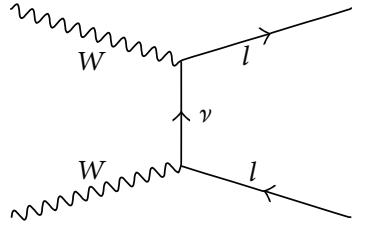

(f)

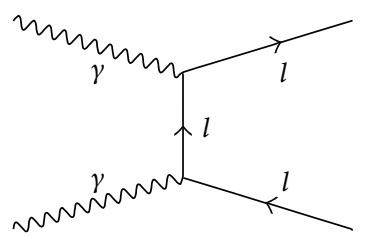

(c)

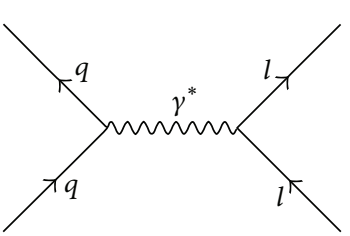

(d)

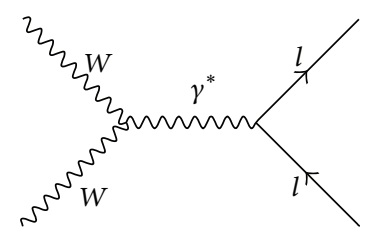

(g)

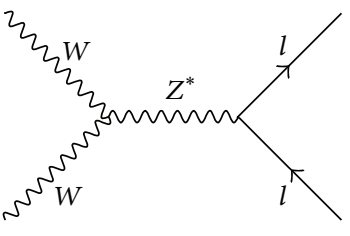

(h)

FIgURE 4: The subprocess Feynman diagrams for dilepton channel.

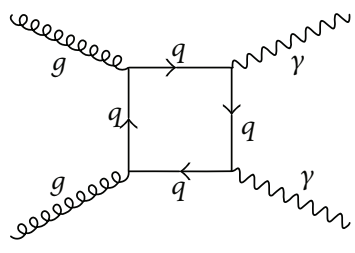

(a)

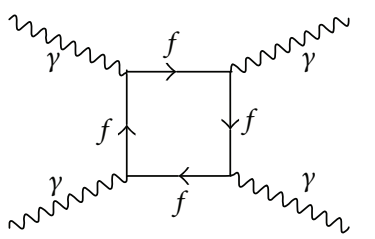

(e)

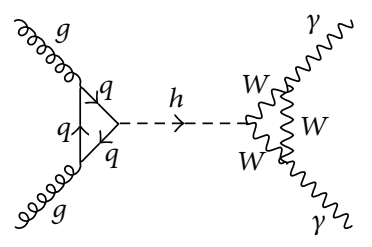

(b)

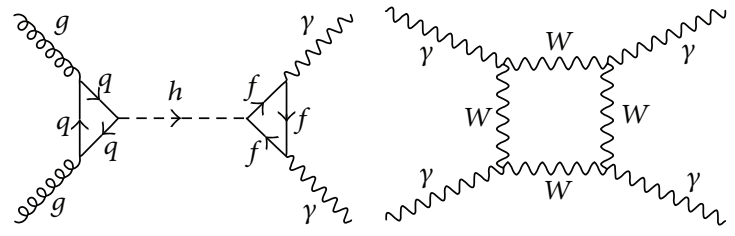

(c)

(d)

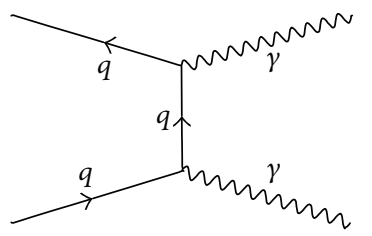

(f)

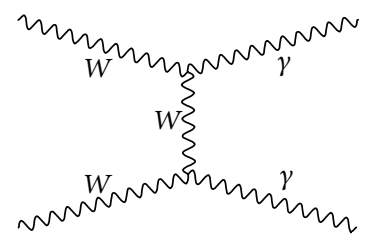

(g)

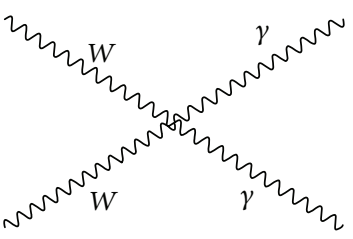

(h)

FIgURE 5: The subprocess Feynman diagrams for diphoton channel.

those reducible backgrounds as these are expected to be very small. Using the Landau-Yang theorem, dual considerations of angular momentum conservation and Bose symmetry prevent a pair of gluons couple to an on-shell $\gamma / \mathrm{Z}$ (via a quark loop, see Figures 4(a) and 4(b)) or a $W W$ pair couple to an on-shell $\gamma / \mathrm{Z}$ (see Figures $4(\mathrm{~g})$ and $4(\mathrm{~h})$ ). While such a coupling, although very small, is indeed permitted for an offshell $\gamma / Z$. The pieces in the corresponding vertex function are proportional to the degree of virtuality of the initial particles or to the momentum of the $\gamma / \mathrm{Z}$ itself. Moreover, $g g \rightarrow l l$ processes involve a quark loop, and $W W \rightarrow l l$ processes are suppressed due to very low $W W$ luminosity. Because of these reasons, we are not including $g g$ and $W W$ initiated processes in our computation. We also neglect $q q$ initiated processes in Figures 4(d) and 4(e) due to very low luminosity for qq. Consequently, these processes lead only to subdominant contributions.

The dominant SM background for the dilepton channel comes from the inelastic photoproduction of the lepton pair as shown in Figure 4(c) where photons are emitted from the quarks inside protons. This contribution can be very large in the kinematic region where the invariant mass of the lepton pair is small. This is because the collinear emission of equivalent photons from quark lines can give large contribution. The inelastic $p p \rightarrow l l$ cross-section has been computed in [59] for the Weizsäcker-Williams photon pairs, and an outline of the computation is presented in Appendix C. The contributions from the interference terms among various background processes might be important at low energies. Since we are only interested in the high energy regions, we do not include them for simplicity.

5.2. Diphoton Backgrounds. In Figure 5, we show possible background subprocesses for the diphoton channel initiated from $g g, \gamma \gamma, q q$, and $W W$ initial states. The dominant background for the diphoton channel comes from the $g g \rightarrow \gamma \gamma$ box diagram (via quark loop) shown in Figure 5(a), and crosssection is proportional to $\alpha_{S}^{2} \alpha_{e m}^{2}$ ( $\alpha_{e m}$ is the QED coupling). There are other higher order diagrams that contribute to $g g \rightarrow \gamma \gamma$ process as shown in Figures 5(b) and 5(c) where Higgs is involved. The Higgs is produced from $g g$ fusion via a top quark loop and then decayed to $\gamma \gamma$ via another top quark or a $W$ loop. There are also Delbruck type (light-bylight scattering) backgrounds possible shown in Figures 5(d) and $5(\mathrm{e})$. In this case, quarks from the dissociated protons 


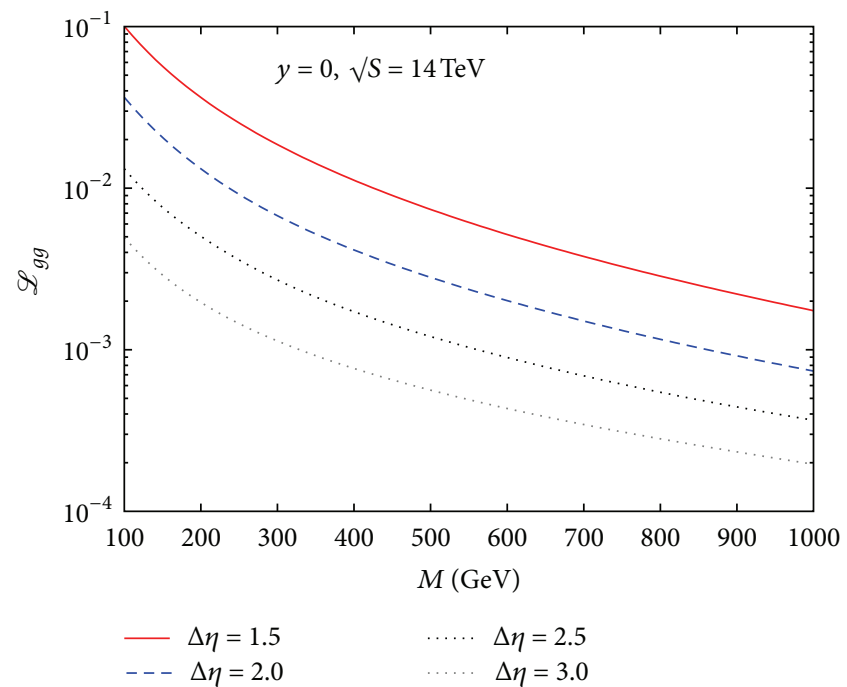

(a)

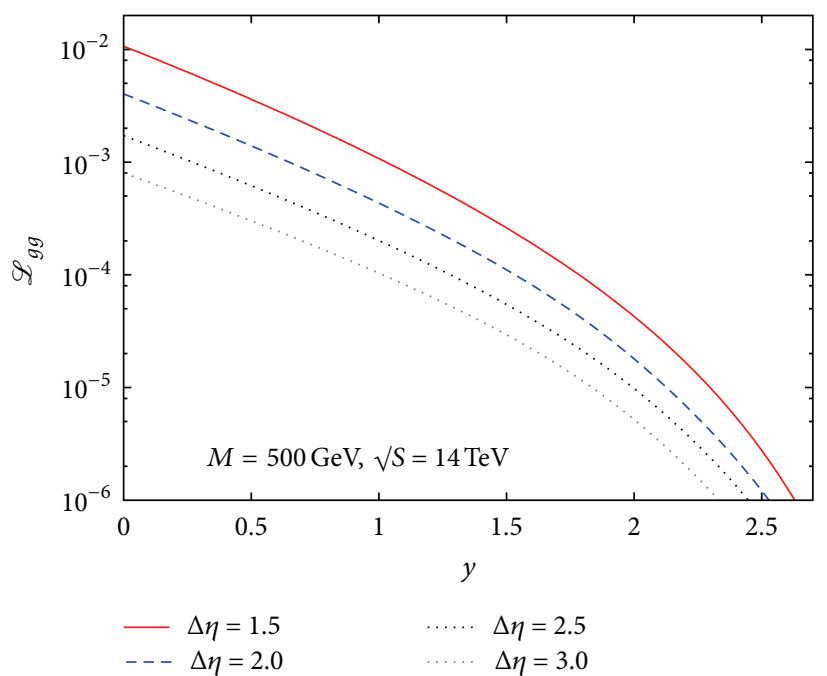

(b)

Figure 6: (a) The effective luminosity $\mathscr{L}_{g g}$ as a function of the invariant mass $M$ of the central system produced at $y=0$ at the 14 TeV LHC and (b) $\mathscr{L}_{g g}$ as a function of $y$ for the production of a system with mass $M=500 \mathrm{GeV}$ at the $14 \mathrm{TeV}$ LHC. For both plots we choose four values of the rapidity gap, that is, $\Delta \eta=1.5,2.0,2.5$, and 3.0.

radiate two photons which then give two final state photons via a fermion or $W$ in the loop. This contribution is much suppressed as cross-section is proportional to $\alpha_{e m}^{4}$. The other backgrounds which are initiated from $q q$ (see Figure 5(f)) and $W W$ (see Figures 5(g) and 5(h)) initial states are negligible as the corresponding effective luminosities are very low. We note that all the $g g$ and $\gamma \gamma$ initiated background processes occur only via loop diagrams, and they are usually very small. If the signal rate is substantial in the diphoton channel, searching the signatures of extra dimensions in the diphoton channel might be very promising.

We estimate the dominant $g g \rightarrow \gamma \gamma$ box diagram background cross-section using $\gamma \gamma \rightarrow \gamma \gamma$ helicity amplitudes taken from [60]. Relevant formulae are given in Appendix D. At low energy, the fermion loop contribution in the box diagram dominates, but above a few hundred $\mathrm{GeV}$, this contribution starts falling rapidly, whereas the $W$ loop contribution dominates in the total cross-section of the $\gamma \gamma \rightarrow \gamma \gamma$ process at high energies. Other higher order $\gamma \gamma \rightarrow$ $h \rightarrow \gamma \gamma$ processes similar to Figures 5(b) and 5(c) are not considered here. We do not include $9 q$ initiated process in our background computation as the contribution originates from $q \bar{q} t$-channel exchange in Figure 5(f) is roughly two order of magnitude lower than the $g g$ contributions [58]. As mentioned earlier, we neglect $W W$ initiated processes for low $W W$ luminosity and all background interference terms for simplicity.

\section{Numerical Results}

In all our numerical computations, we use the MSTW2008 LO parton densities to obtain the unintegrated parton distributions. Here, we once again give the numerical values of some quantities which are used. To compute the Sudakov form factors, we use NLO $\alpha_{S}$ with $\Lambda_{\mathrm{QCD}}=220 \mathrm{MeV}$ and number of "active" flavor $N_{f}=5$. We take a fixed value of the "gap survival factor," $\mathcal{S}^{2}=0.1$ in our analysis. Our results depend on two model parameters, the number of extra dimensions $n$ and the ultraviolet cutoff $M_{S}$ of the theory, and four kinematical variables, invariant mass $M$ and rapidity $y$ of the centrally produced system, rapidity gap $\Delta \eta$ between the central system and the outgoing protons or proton dissociated products, and the CM energy $\sqrt{S}$ of two colliding protons at the LHC. The cutoff scale $M_{S}$ is of the order of few TeV for low scale gravity theories. The lower limit of $M_{S}$ is bounded from experiments, and the bounds are already quite high as stated in Section 2. If we increase $M_{S}$, the signal crosssections decrease as usual. In our case, the signal falls off very rapidly with increasing $M_{S}$ because of the presence of $M_{S}^{8}$ in the denominator of the signal cross-sections (see (12) and (16)). This makes the ADD model very difficult to search for experimentally.

In Figure 6(a), we show the dependence of the effective luminosity $\mathscr{L}_{g g}$ on the invariant mass $M$ of the central system produced at rapidity region $y=0$ at the $14 \mathrm{TeV} \mathrm{LHC}$. The $\mathscr{L}_{g g}$ shows a decreasing nature with increasing $M$ since it is harder to produce more massive objects while keeping other parameters fixed. In Figure 6(b), we plot the dependence of $\mathscr{L}_{g g}$ on $y$ with $M=500 \mathrm{GeV}$ at the $14 \mathrm{TeV}$ LHC. In the diffractive processes, it is more likely to produce an object centrally, that is, near $y=0$ region. Thus, a system which is produced away from the central region is less probable, and we see a decreasing behavior of $\mathscr{L}_{g g}$ with increasing $y$.

In Figure 7, we show the total signal cross-section in the dilepton $\left(\sigma_{l l}\right.$ where $\left.l=\{e, \mu\}\right)$ channel as a function of $\sqrt{S}$. In Figure $7(\mathrm{a})$, we show $\sigma_{l l}$ for different $\Delta \eta$ taking $n=2$ and $M_{S}=2 \mathrm{TeV}$. The cross-section increases as we increase $\sqrt{S}$ as expected. If we increase $\Delta \eta$, the $\sigma_{l l}$ decreases as 


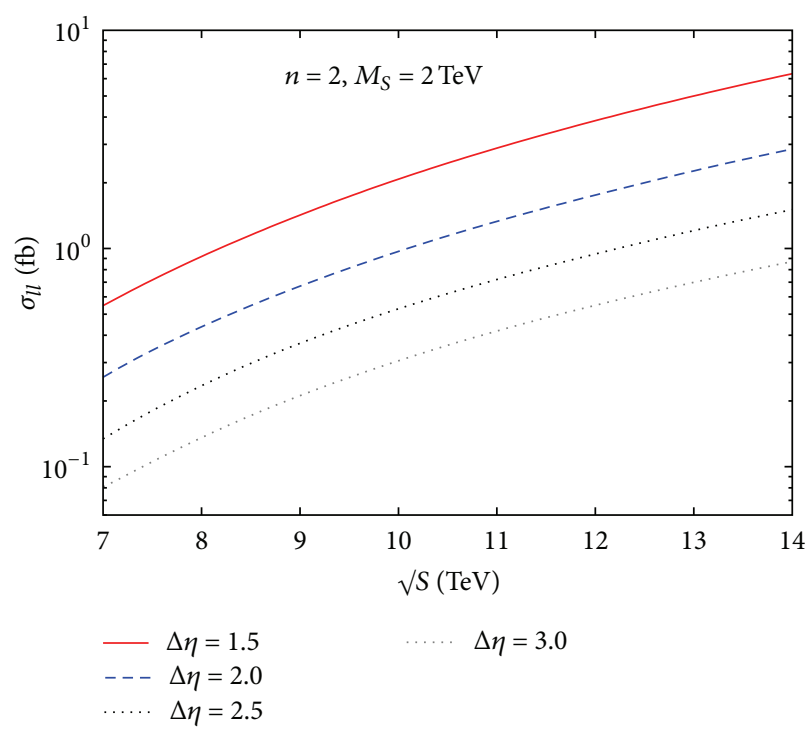

(a)

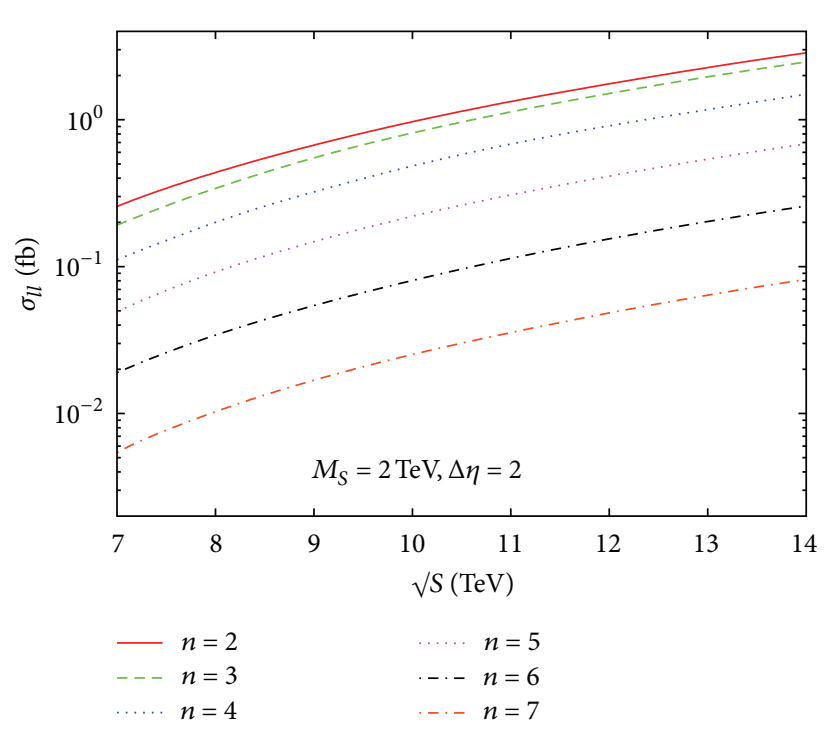

(b)

FIGURE 7: The total signal cross-section $(p p \rightarrow X+l l+Y)$ in dilepton channel $\left(\sigma_{l l}\right)$ as a function of LHC CM energy. In (a), we plot $\sigma_{l l}$ for $\Delta \eta=1.5,2.0,2.5$, and 3.0 for $n=2$ and $M_{S}=2 \mathrm{TeV}$. In (b), we plot $\sigma_{l l}$ for $n=2-7$ for $M_{S}=2 \mathrm{TeV}$ and $\Delta \eta=2$. In both plots, $\sigma_{l l}$ 's are computed after applying the cut defined in (17).

the effective luminosity decreases with increasing $\Delta \eta$ (see Figures 6(a) and 6(b)). In Figure 7(b), we show the $\sigma_{l l}$ for different $n$ varied from two to seven. We see that as we increase $n$, the $\sigma_{l l}$ decreases. This is because $\kappa^{2}\left|\mathscr{D}_{\text {eff }}\right| \sim 2 /(n-$ 2) for $n>2$, in the limit $M_{S}^{2} \gg \widehat{s}$ (see (B.9)). Here, we compute the total cross-section after applying the following selection cuts:

$$
\begin{gathered}
\left|y_{\psi \psi}\right| \leq 2, \quad\left|\cos \theta_{\psi \psi}\right| \leq 1, \\
100 \mathrm{GeV} \leq M_{\psi \psi}<M_{S}-10 \mathrm{GeV},
\end{gathered}
$$

where $\theta$ is the scattering angle of $\psi$ (where $\psi=l, \gamma$ ) in the rest frame of the centrally produced system and $M_{\psi \psi}$ is the invariant mass of the $\psi \psi$ pair. We have collected events with the rapidity of the central system $\left|y_{\psi \psi}\right| \leq 2$. The upper limit of $M_{\psi \psi}$ can go to $M_{S}$ up to which the theory is valid. Here, we have taken the upper limit on $M_{\psi \psi}$ slightly smaller than $M_{S}$ as the $I$ function in (9) diverges at $M_{S}$. The cross-sections are only few $f b$, and we need higher CM energy at the LHC to observe these events.

In Figure 8, we show the total signal cross-section in the dilepton $\left(\sigma_{l l}\right)$ channel as a function of $M_{S}$ at the $14 \mathrm{TeV}$ LHC. In Figure 8(a), we show $\sigma_{l l}$ for different $\Delta \eta$ taking $n=2$, and in Figure 8(b), we show $\sigma_{l l}$ for different $n$ taking $\Delta \eta=2$. Here, we compute the total cross-section after applying the kinematical cuts defined in (17). The crosssection decreases rapidly as we increase $M_{S}$ since $M_{S}^{8}$ is present in the denominator of the total cross-section.

In Figure 9(a), we show signal and background invariant mass distributions $\left(d \sigma_{l l} / d M_{l l}\right)$ of the lepton pair after applying the selection cuts defined in (18) at the $14 \mathrm{TeV}$ LHC. To compute $d \sigma_{l l} / d M_{l l}$, we have not integrated $\cos \theta_{\psi \psi}$ in the full range from -1 to 1 because the background crosssection diverges as $\left|\cos \theta_{\psi \psi}\right| \rightarrow 1$ (see (C.5)). We plot signal $d \sigma_{l l} / d M_{l l}$ for $M_{S}=1.5,2.0$, and $2.5 \mathrm{TeV}$ taking $n=2$ and $\Delta \eta=2$ for both signal and background. The signal $d \sigma_{l l} / d M_{l l}$ is a monotonically increasing function of $M_{l l}\left(M_{l l}^{2}=\widehat{s}\right)$ because the subprocess cross-section increases roughly as $\widehat{s}^{3}$ (see (12)). The background cross-section is computed only considering the dominant $\gamma \gamma \rightarrow l l$ channel. For small values of $M_{l l}$, the background is quite large but falls off rapidly as we increase $M_{l l}$. Thus, to observe an excess over the background one should collect lepton pairs with sufficiently high $M_{l l}$ :

$$
\left|y_{l l}\right| \leq 2, \quad\left|\cos \theta_{l l}\right| \leq 0.8
$$

In Figure 9(b), we show signal and background angular distributions $\left(d \sigma_{l l} / d \cos \theta_{l l}\right)$ of the lepton pair after applying the selection cuts defined in (19) at the $14 \mathrm{TeV}$ LHC. To compute $d \sigma_{l l} / d \cos \theta_{l l}$, we apply a very high invariant mass cut on the lepton pair to reduce large background in the small $M_{l l}$ region. We show signal $d \sigma_{l l} / d \cos \theta_{l l}$ for $M_{S}=1.5,2.0$, and $2.5 \mathrm{TeV}$ taking $n=2$ and $\Delta \eta=2$ for both signal and background. The angular distribution carries spin information of the intermediate particle. For dilepton production, the angular distributions for signal and background show a very contrasting behavior. For signal $d \sigma_{l l} / d \cos \theta_{l l} \sim\left(1-\cos ^{4} \theta_{l l}\right)$ (see (12)) which attains its maximum at $\cos \theta_{l l}=0$. On the other hand, background attains its minimum at $\cos \theta_{l l}=$ 0 since it goes as $\left(1+\cos ^{2} \theta_{l l}\right) / \sin ^{2} \theta_{l l}$ and diverges when $\left|\cos \theta_{l l}\right| \rightarrow 1$ (see (C.5)). This contrasting feature can be used as a unique signature of the extra dimensions

$$
\left|y_{l l}\right| \leq 2, \quad 750 \mathrm{GeV} \leq M_{l l}<1250 \mathrm{GeV} .
$$




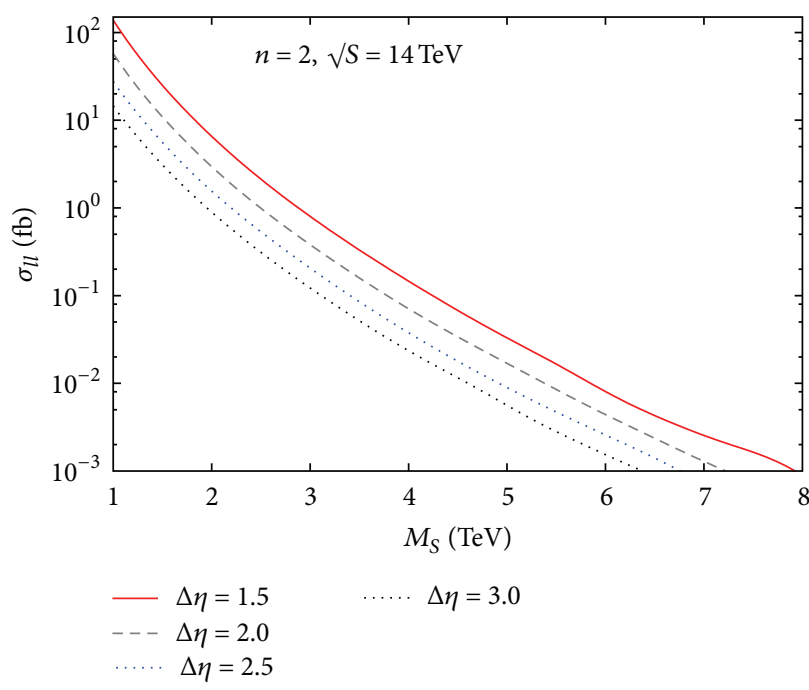

(a)

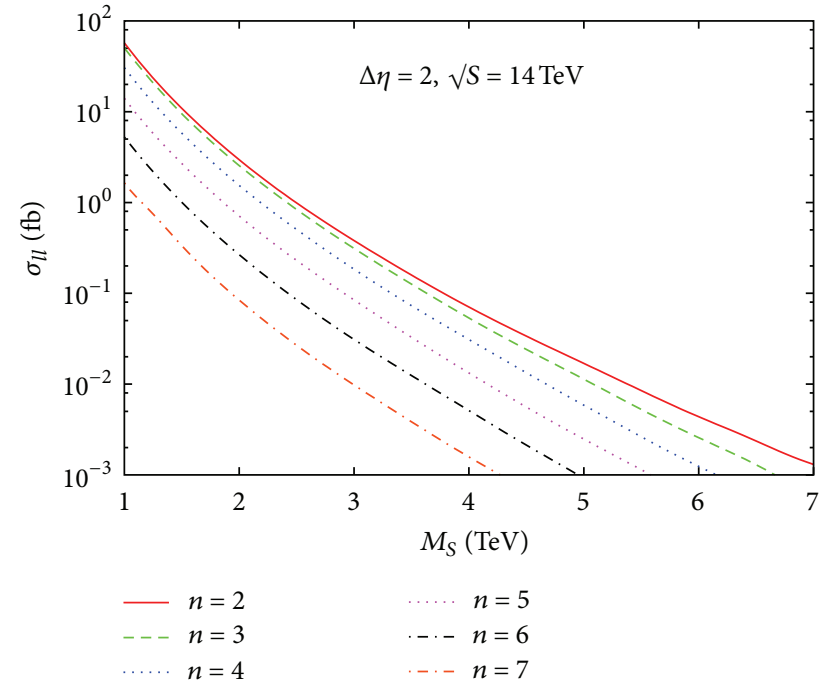

(b)

FIGURE 8: The total signal cross-sections $\left(p p \rightarrow X+l l+Y\right.$ ) in dilepton channel $\left(\sigma_{l l}\right)$ as a function of $M_{S}$ at the $14 \mathrm{TeV}$ LHC. In (a), we plot $\sigma_{l l}$ for $\Delta \eta=1.5,2.0,2.5$, and 3.0 for $n=2$. In (b), we plot $\sigma_{l l}$ for $n=2-7$ for $\Delta \eta=2$. In both plots, $\sigma_{l l}$ 's are computed after applying the cut defined in (17).

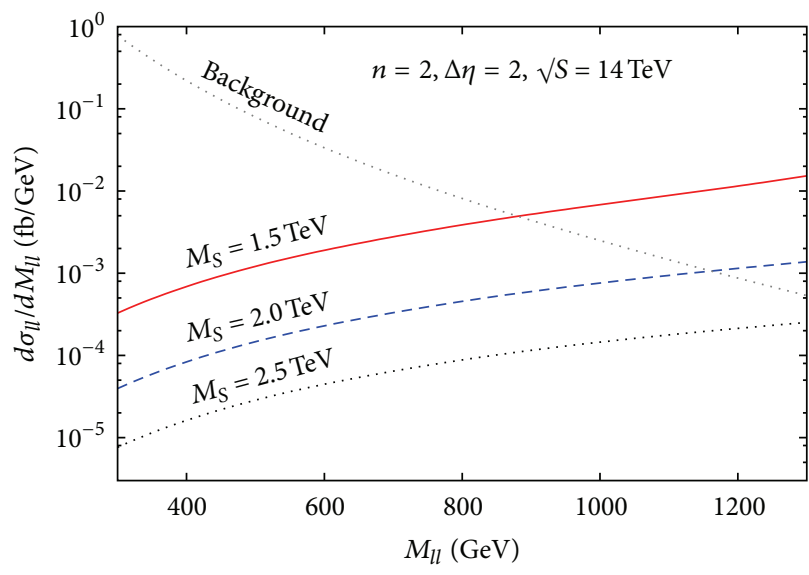

(a)

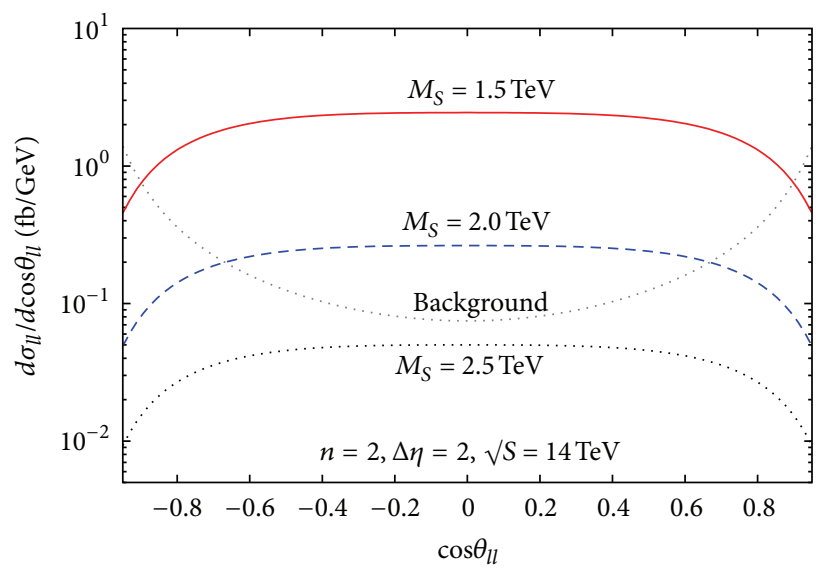

(b)

Figure 9: (a) The invariant mass distributions $\left(d \sigma_{l l} / d M_{l l}\right)$ for signal and background $(p p \rightarrow X+l l+Y)$ of the lepton pair after applying the cuts defined in (18) at the $14 \mathrm{TeV}$ LHC. (b) The angular distributions $\left(d \sigma_{l l} / d \cos \theta_{l l}\right)$ for signal and background of the lepton pair after applying the cuts defined in (19) at the $14 \mathrm{TeV}$ LHC. For both plots, we take $M_{S}=1.5,2.0$, and $2.5 \mathrm{TeV}$ and $n=2$ for signal and $\Delta \eta=2$ for both signal and background.

In Figure 10, we show the total signal cross-section in the diphoton channel $\left(\sigma_{\gamma \gamma}\right)$ as a function of $\sqrt{S}$ after applying the cut defined in (17). In Figure 10(a), we show $\sigma_{\gamma \gamma}$ for different $\Delta \eta$ taking $n=2$ and $M_{S}=2 \mathrm{TeV}$. In Figure 10(b), we show $\sigma_{\gamma \gamma}$ for different $n$ varied from two to seven. In Figure 11, we show the $\sigma_{\gamma \gamma}$ as a function of $M_{S}$ at the $14 \mathrm{TeV}$ LHC. In Figure 11(a), we show $\sigma_{\gamma \gamma}$ for different $\Delta \eta$ taking $n=2$, and in Figure 11(b), we show $\sigma_{\gamma \gamma}$ for different $n$ taking $\Delta \eta=2$. Here, we compute the $\sigma_{\gamma \gamma}$ after applying the kinematical cuts defined in (17). The behavior of these plots is very similar to the corresponding plots for dilepton channel, and we are not repeating those features here. An interesting point to notice is that $\sigma_{l l}$ and $\sigma_{\gamma \gamma}$ are similar even quantitatively. This is because surprisingly $\sigma_{l l}$ and $\sigma_{\gamma \gamma}$ are equal after $\cos \theta$ integration from -1 to 1 as follows:

$$
\begin{aligned}
\sum_{l=e, \mu} \int_{-1}^{1} d(\cos \theta) \frac{d \widehat{\sigma}\left(g g \rightarrow l^{+} l^{-}\right)}{d|\cos \theta|} \\
\quad=\int_{-1}^{1} d(\cos \theta) \frac{d \widehat{\sigma}(g g \rightarrow \gamma \gamma)}{d|\cos \theta|} .
\end{aligned}
$$




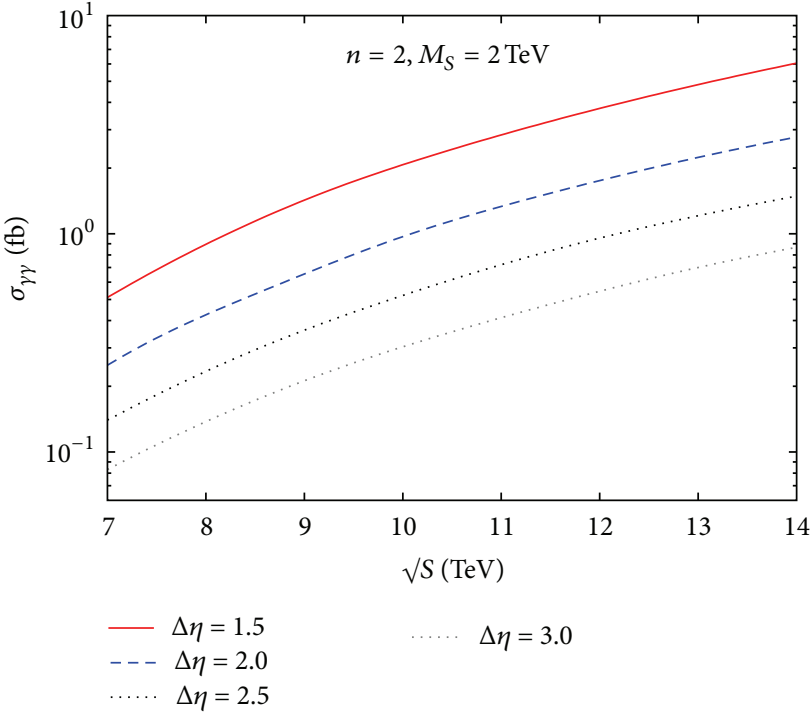

(a)

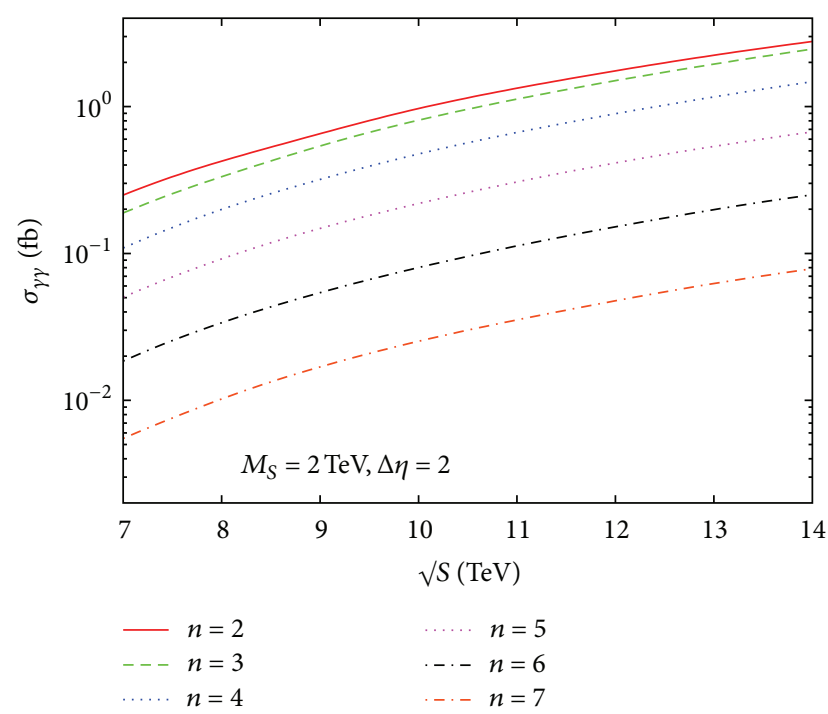

(b)

FIgURE 10: The total signal cross-sections $(p p \rightarrow X+\gamma \gamma+Y)$ in diphoton channel $\left(\sigma_{l l}\right)$ as a function of LHC CM energy. In (a), we plot $\sigma_{l l}$ for $\Delta \eta=1.5,2.0,2.5$, and 3.0 for $n=2$ and $M_{S}=2 \mathrm{TeV}$. In (b), we plot $\sigma_{l l}$ for $n=2-7$ for $M_{S}=2 \mathrm{TeV}$ and $\Delta \eta=2$. In both plots, $\sigma_{l l}$ 's are computed after applying the cut defined in (17).

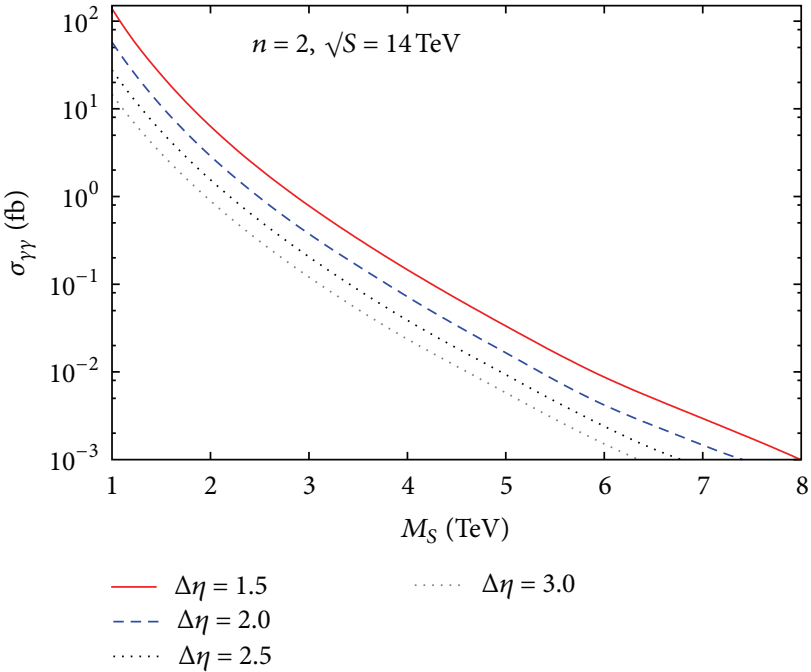

(a)

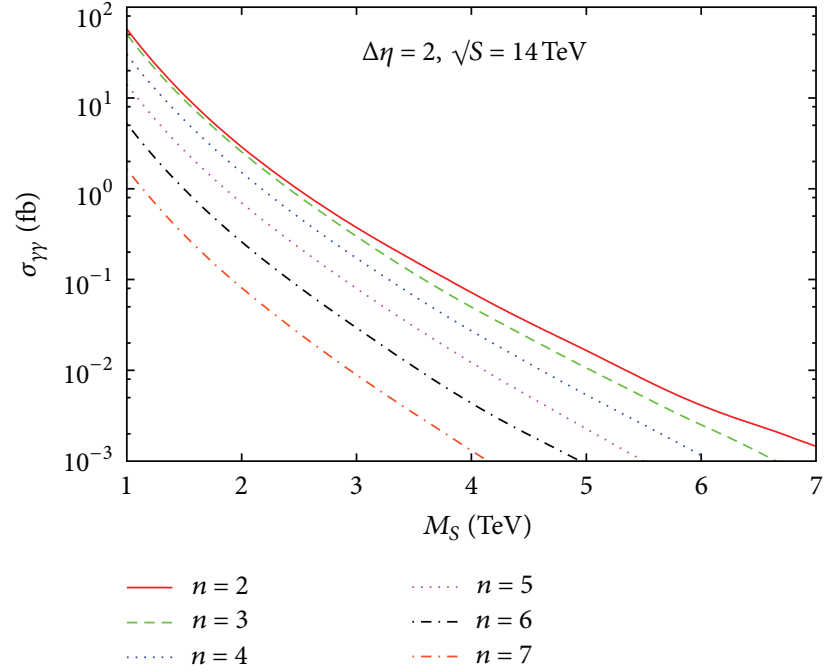

(b)

Figure 11: The total signal cross-sections ( $p p \rightarrow X+\gamma \gamma+Y$ ) in diphoton channel $\left(\sigma_{\gamma \gamma}\right)$ as a function of $M_{S}$ at the $14 \mathrm{TeV}$ LHC. In (a), we plot $\sigma_{\gamma \gamma}$ for $\Delta \eta=1.5,2.0,2.5$, and 3.0 for $n=2$. In (b), we plot $\sigma_{\gamma \gamma}$ for $n=2-7$ for $\Delta \eta=2$. In both plots, $\sigma_{\gamma \gamma}$ 's are computed after applying the cut defined in (17).

In Figure 12(a), we show signal and background invariant mass distributions $\left(d \sigma_{\gamma \gamma} / d M_{\gamma \gamma}\right)$ of the diphoton pair after applying the selection cuts defined in (21) at the $14 \mathrm{TeV}$ LHC:

$$
\left|y_{\gamma \gamma}\right| \leq 2, \quad\left|\cos \theta_{\gamma \gamma}\right| \leq 1 .
$$

We plot signal $d \sigma_{\gamma \gamma} / d M_{\gamma \gamma}$ for $M_{S}=1.5,2.0$, and $2.5 \mathrm{TeV}$ taking $n=2$ and $\Delta \eta=2$ both for signal and background. The signal $d \sigma_{\gamma \gamma} / d M_{\gamma \gamma}$ is a monotonically increasing function of $M_{\gamma \gamma}\left(M_{\gamma \gamma}^{2}=\widehat{s}\right)$ because the subprocess crosssection increases roughly as $\widehat{s}^{3}$ (see (16)). The background cross-section is computed including $g g$ and $\gamma \gamma$ initiated processes as shown in Figure 5. Although the background in Figure 12(a) can beat signal in the small $M_{\gamma \gamma}$ region, it dies out very rapidly in the high $M_{\gamma \gamma}$ region. This is because the diphoton background goes roughly as $1 / M_{\gamma \gamma}^{2}$ and becomes almost negligible at large $M_{\gamma \gamma}$. In Figure 12(b), we show signal and background angular distributions $\left(d \sigma_{\gamma \gamma} / d \cos \theta_{\gamma \gamma}\right)$ of the diphoton pair after applying the selection cuts defined in (22) at the $14 \mathrm{TeV}$ LHC:

$$
\left|y_{\gamma \gamma}\right| \leq 2, \quad 250 \mathrm{GeV} \leq M_{\gamma \gamma}<1250 \mathrm{GeV} .
$$




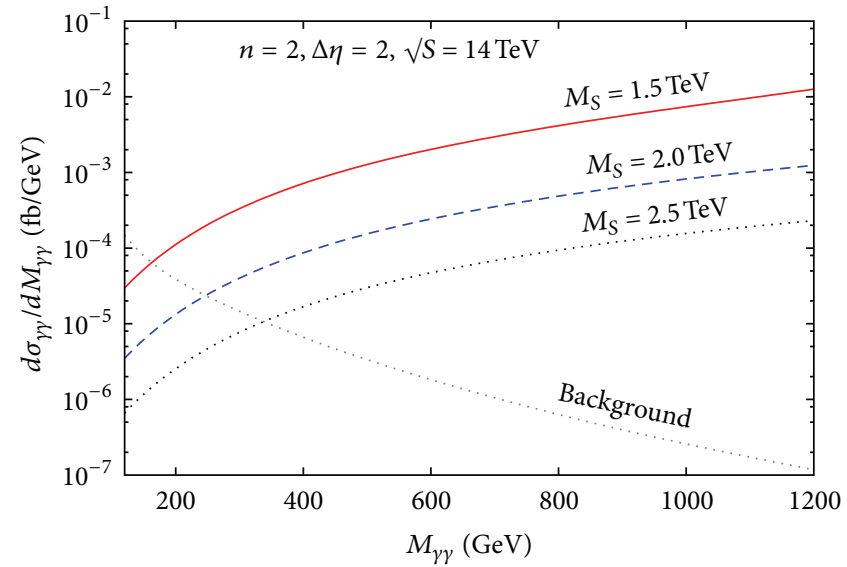

(a)

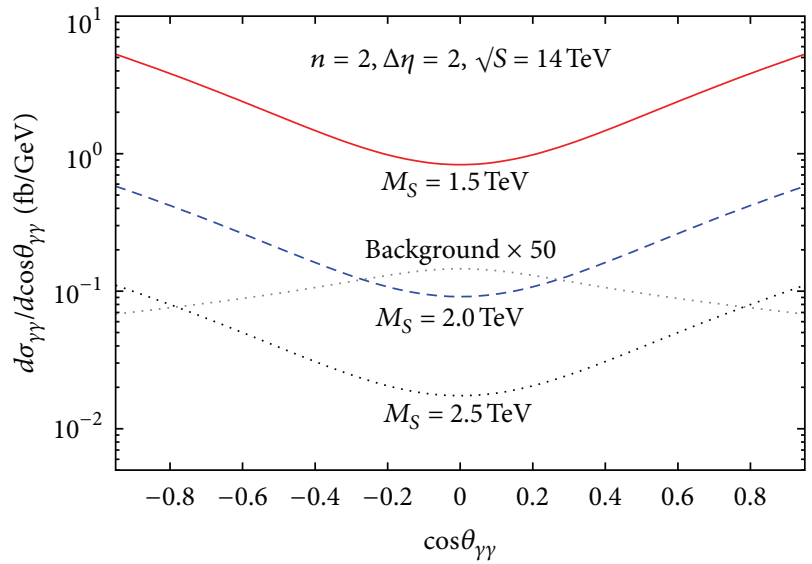

(b)

FiguRE 12: (a) The invariant mass distributions $\left(d \sigma_{\gamma \gamma} / d M_{\gamma \gamma}\right)$ for signal and background $(p p \rightarrow X+\gamma \gamma+Y)$ of the photon pair after applying the cuts defined in (18) at the $14 \mathrm{TeV}$ LHC. (b) The angular distributions $\left(d \sigma_{\gamma \gamma} / d \cos \theta_{\gamma \gamma}\right)$ for signal and background after applying the cuts defined in (19) at the $14 \mathrm{TeV}$ LHC. For both plots, we take $M_{S}=1.5,2.0$, and $2.5 \mathrm{TeV}$ and $n=2$ for signal and $\Delta \eta=2$ for both signal and background. The background in (b) is shown after multiplying by a factor of 50 .

We see that diphoton signal and background angular distributions behave just in the opposite way to the dilepton signal and background angular distributions, respectively. The signal $d \sigma_{\gamma \gamma} / d \cos \theta_{\gamma \gamma} \sim\left(1+6 \cos ^{2} \theta+\cos ^{4} \theta\right)$ has a minimum at $\cos \theta=0$ and attains its maximum value at $|\cos \theta|=1$, whereas the $d \sigma_{\gamma \gamma} / d \cos \theta_{\gamma \gamma}$ for the dominant $g g \rightarrow \gamma \gamma$ background attains its maximum value at $\cos \theta=$ 0 as shown in Figure 12(b). This is because of the nature of angular dependent part in (D.1). The similar angular behavior is true for subdominant $\gamma \gamma \rightarrow \gamma \gamma$ background also. Whereas, there is no angular dependency of other subdominant $g g \rightarrow h \rightarrow \gamma \gamma$ backgrounds because of the spin-0 nature of the intermediate Higgs.

6.1. LHC Discovery Potential. We define the luminosity requirement for the discovery of KK gravitons at the LHC as follows:

$$
L_{D}=\operatorname{Max}\left\{L_{5}, L_{10}\right\} \text {, }
$$

where $L_{5}$ denotes the luminosity required to attain $5 \sigma$ statistical significance and $L_{10}$ is the luminosity required to observe 10 signal events. We compute $L_{D}$ after applying some kinematical cuts which we call the "discovery cuts" as defined in the following.

6.1.1. Discovery Cuts. Motivated by the invariant mass and angular distributions in Figures 9 and 12, we construct some kinematical cuts to separate the signal from the background in the dilepton and diphoton channels as follows:

(1) dilepton channel: $\left|y_{l l}\right| \leq 2 ;\left|\cos \theta_{l l}\right| \leq 0.8 ; M_{S} / 2 \leq$ $M_{l l}<M_{S}-10 \mathrm{GeV}$;

(2) diphoton channel: $\left|y_{\gamma \gamma}\right| \leq 2 ;\left|\cos \theta_{\gamma \gamma}\right| \leq 1 ; 250 \mathrm{GeV} \leq$ $M_{\gamma \gamma}<M_{S}-10 \mathrm{GeV}$.

We call these cuts as the "discovery cuts" for the discovery of KK graviton at the LHC. In Figure $13, L_{D}$ goes as $L_{10}$ for both dilepton and diphoton channels in the whole parameter space we have displayed. In case of the diphoton channel, the background is already quite small compared to the signal, whereas for the dilepton channel, background becomes under control after the "discovery cuts." With the "discovery cuts," one can probe $M_{S}$ roughly up to $3.6 \mathrm{TeV}(4.4 \mathrm{TeV})$ with $100 \mathrm{fb}^{-1}\left(300 \mathrm{fb}^{-1}\right)$ integrated luminosity at the $14 \mathrm{TeV}$ LHC.

\section{Conclusions}

In this paper, we have discussed the possibility of looking for new physics, in particular, the signatures of large extra dimensions, in diffractive processes at the LHC. We have concentrated on the central inclusive production where the outgoing protons breakup, but the fragments are mostly emitted in the forward direction. We have studied in detail the signal of KK gravitons via the dilepton and the diphoton channels. From angular momentum conservation, the spin2 graviton cannot be produced at LO in exclusive processes as discussed in the text, but it can be produced in an inclusive configuration. Although the backgrounds are larger for inclusive production, the larger cross-section and event rate for signals make the collider search for extra dimensional theories very promising. Since the intermediate particle is spin-2 in nature, we also get a unique angular distribution which can be used to distinguish signals from backgrounds. We have shown that it is very unlikely to produce a lepton or a photon pair in the SM with very high invariant mass in the inclusive configuration. Thus, experimentally, if we find a reasonable number of lepton or photon pairs with high invariant mass, it would clearly indicate the signature of new physics possibly the signature of large extra dimensions.

In this work, we have indicated the possibility of using dilepton and diphoton in the final state to signal the presence of extra dimensions. We have carefully estimated the sizes of the signal and the background and found that the signal rates are not too big. Nevertheless, it is possible within reasonable parameter ranges as suggested by present day studies to use central production, with forward detectors for the proton 


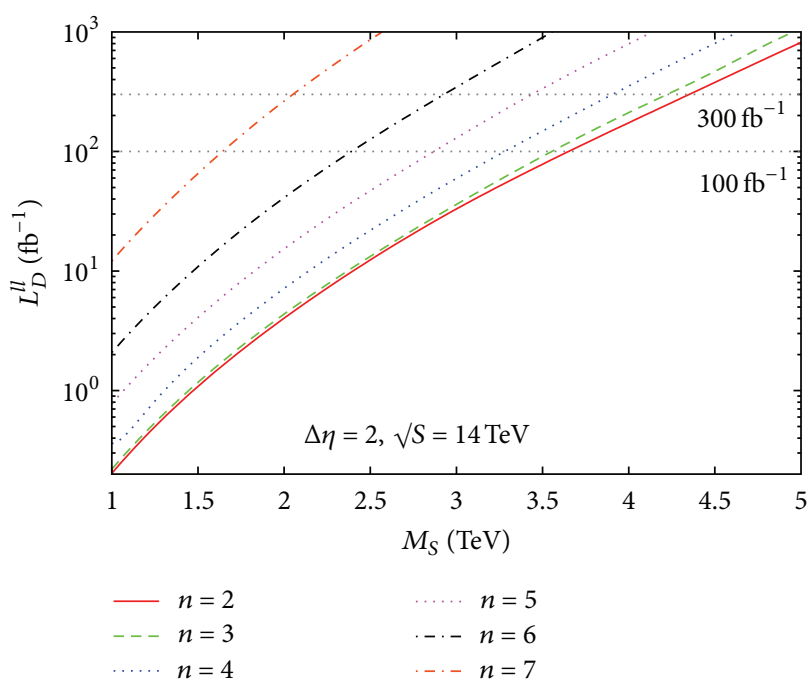

(a)

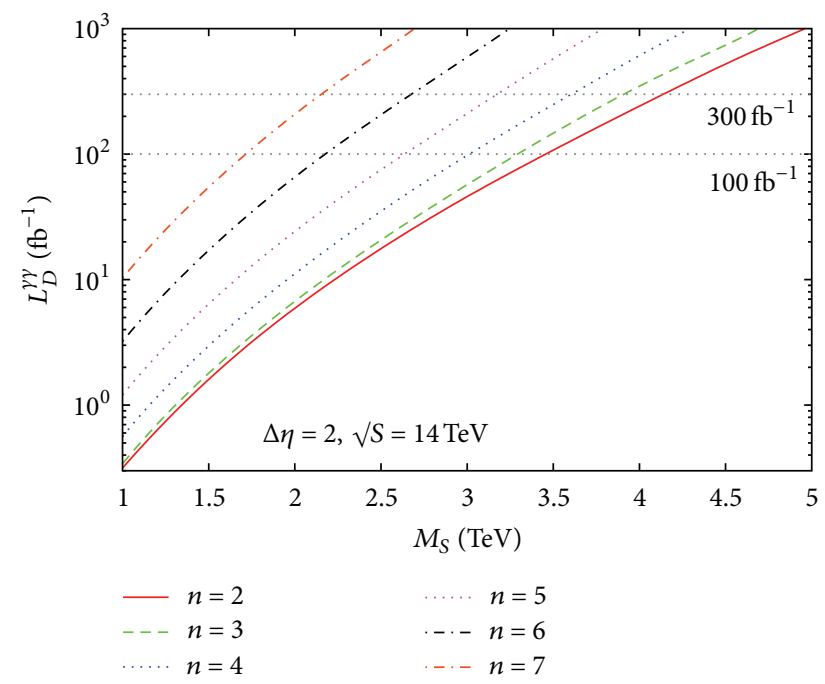

(b)

Figure 13: The required luminosity $\left(L_{D}\right)$ for the discovery of KK graviton in (a) dilepton and (b) diphoton channels as a function of $M_{S}$ at the $14 \mathrm{TeV}$ LHC with "discovery cuts" (see text for the definitions of the cuts). The $L_{D}^{l l}$ and $L_{D}^{\gamma \gamma}$ are computed for $n=2-7$ with $\Delta \eta=2$ at $p p \rightarrow X+\psi \psi+Y$ level where $\psi=\{l, \gamma\}$.

fragments, to look for KK gravitons as a signal for ADD-like theories. The great advantage of using such channels for the study of new physics is that they are clean, and the signals are easily distinguished from the backgrounds. On the flip side, the event rates are considerably smaller than other standard new physics searches. This can be compensated by runs at higher energies and greater luminosities at some future date. There is another general issue with high luminosity run at the LHC-the presence of huge "pile-up" background. This background can overwhelm the rapidity gap signatures. If we consider inclusive processes and sacrifice the forward proton tagging (applicable only for exclusive processes), we can detect rapidity gap events at the LHC [8].

All higher dimensional models are valid up to a cutoff scale. In our case, the signal cross-sections depend on the eighth power of $1 / M_{S}\left(M_{S}\right.$ is the cutoff scale of the theory). Thus, numerical results are very sensitive to the UV cutoff. The experimental lower bound of $M_{S} \approx 3 \mathrm{TeV}$ for $n=2$. Future experiments can push up the lower bound and make the signatures less visible. We show that LHC with $\sqrt{S}=$ $14 \mathrm{TeV}$ and $100 \mathrm{fb}^{-1}\left(300 \mathrm{fb}^{-1}\right)$ of integrated luminosity can probe $M_{S}$ up to $3.6 \mathrm{TeV}(4.4 \mathrm{TeV})$ in both the dilepton and the diphoton channels.

\section{Appendices}

\section{A. Inclusive Luminosity}

An outline of the derivation of effective luminosity for the inclusive configuration is presented in [32]. Here, we briefly give some useful formulae for computing inclusive luminosity. At the partonic level, the inclusive process in Figure 14 can be expressed as the exclusive production of the system $M$ with rapidity gaps on either side, that is,

$$
a_{1} a_{2} \longrightarrow a_{1}+M+a_{2} .
$$

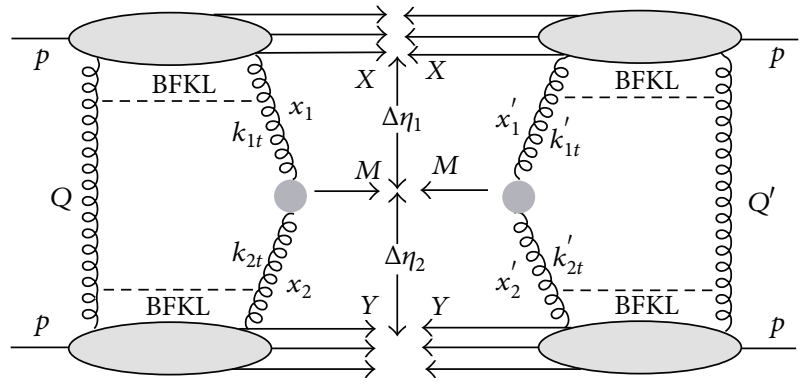

FIGURE 14: The inclusive double diffractive amplitude multiplied by its complex conjugate. A central system $M$ is produced with rapidity gaps $\Delta \eta_{1}$ and $\Delta \eta_{2}$ between proton dissociated products $X$ and $Y$, respectively.

The probabilities to find partons $a_{i}(i=\{1,2\})$ in the protons is given by the effective parton densities $\mathscr{G}\left(x_{i}, k_{i t}^{2}\right)$ evaluated at scale $k_{i t}^{2}$ with momentum fraction $x_{i}$ as given by

$$
\begin{aligned}
\mathscr{G}\left(x_{i}, k_{i t}^{2}\right)= & x_{i} g\left(x_{i}, k_{i t}^{2}\right) \\
& +\frac{16}{81} \sum_{q} x_{i}\left[q\left(x_{i}, k_{i t}^{2}\right)+\bar{q}\left(x_{i}, k_{i t}^{2}\right)\right],
\end{aligned}
$$

where $g, q$, and $\bar{q}$ are the usual PDFs. The rapidity gaps can be filled up due to gluon bremsstrahlung effect. The mean number of gluons emitted in transverse momentum interval, $Q_{t}<P_{t}<k_{i t}$ (also $Q_{t}^{2} \ll k_{i t}^{2}$, asymmetric $t$-channel gluon exchange) with rapidity gap $\Delta \eta_{i}$ is given by

$$
n_{i}=\frac{3 \alpha_{S}}{\pi} \Delta \eta_{i} \ln \left(\frac{k_{i t}^{2}}{Q_{t}^{2}}\right) .
$$

Therefore, the amplitude for no emission in the rapidity interval $\Delta \eta_{i}$ can be expressed as $A_{i}=\exp \left(-n_{i} / 2\right) \Phi\left(Y_{i}\right)$. 
The amplitude $A_{i}$ is called the nonforward BFKL amplitude which is computed resumming the double logarithms in (A.3). The factor $\Phi\left(Y_{i}\right)$ accounts for the longitudinal BFKL logarithm where $Y_{i}=\left(3 \alpha_{S} / 2 \pi\right) \Delta \eta_{i}$. For rapidity gap $\Delta \eta_{i} \lesssim 4$, we have $Y_{i} \lesssim 0.4$. In the asymmetric region, $Q_{t}^{2} \ll k_{i t}^{2}$, it is sufficient to keep only the $\mathcal{O}\left(Y_{i}\right)$ term, that is, $\Phi\left(Y_{i}\right) \approx$ $1+Y_{i} Q_{t}^{2} / k_{i t}^{2} \approx 1.1 \pm 0.1[61]$. There is another suppression that comes in the form of the Sudakov factor $T\left(k_{i t}, \mu\right)$ which accounts for the survival probability of a gluon in the interval $k_{i t}<P_{t}<\mu$ where $\mu$ is related to the hard scale as $\mu=M / 2$. The Sudakov factor reads as

$$
\begin{aligned}
T\left(k_{i t}, \mu\right)=\exp ( & -\int_{k_{i t}^{2}}^{\mu^{2}} \frac{\alpha_{S}\left(k_{t}^{2}\right)}{2 \pi} \frac{d k_{t}^{2}}{k_{t}^{2}} \\
& \left.\times \int_{0}^{1-\Delta}\left[z P_{g g}(z)+\sum_{q} P_{q g}(z)\right] d z\right),
\end{aligned}
$$

where $P_{g g}(z)$ and $P_{q g}(z)$ are the LO DGLAP splitting functions and $\Delta=k_{t} /\left(\mu+k_{t}\right)$. We obtain the effective luminosity $\left(\mathscr{L}_{g g}\right)$ by multiplying the inclusive amplitude by its complex conjugate as shown in Figure 14 . To compute $\mathscr{L}_{g g}$, we carefully account for all the factors, that is, nonforward BFKL amplitudes, the Sudakov factors, gap survival factor, and appropriate color factors, and finally arrive at the expression

$$
\begin{aligned}
\mathscr{L}_{g g}= & \mathcal{S}^{2} \int_{x_{1}^{\min }}^{1} \mathscr{G}\left(x_{1}, k_{1 t}^{2}\right) \frac{d x_{1}}{x_{1}} \\
& \times \int_{x_{2}^{\min }}^{1} \mathscr{G}\left(x_{2}, k_{2 t}^{2}\right) \frac{d x_{2}}{x_{2}} \frac{\alpha_{S}^{4}}{\pi^{2}}\left(\frac{N_{c}^{2}}{N_{c}^{2}-1}\right)^{2} \mathscr{I}_{g g},
\end{aligned}
$$

where

$$
\mathscr{I}_{g g}=\int \frac{d Q_{t}^{2}}{Q_{t}^{2}} \frac{d Q^{\prime 2}}{Q_{t}^{\prime 2}} \frac{d k_{1 t}^{2}}{k_{1 t}^{2}} \frac{d k_{2 t}^{2}}{k_{2 t}^{2}}\left(A_{1} A_{2} A_{1}^{\prime} A_{2}^{\prime}\right) \sqrt{T_{1} T_{2} T_{1}^{\prime} T_{2}^{\prime}}
$$

Here, all the primed quantities are arising from the complex conjugate of the amplitude. The minimum of the momentum fraction $x_{i}^{\mathrm{min}}$ maintains the rapidity gap $\Delta \eta_{i}$ and is given by

$$
x_{i}^{\min }=\frac{M}{\sqrt{S}} \exp (y)+\frac{k_{i t}}{\sqrt{S}} \exp \left(y+\Delta \eta_{i}\right) .
$$

The momentum of the screening gluon $Q$ is very small. Therefore, in the limit $Q^{2} \ll k_{i}^{2}$, we have $t_{i}=\left(Q-k_{i}\right)^{2} \approx$ $-k_{i t}^{2} \approx-k_{i t}^{\prime 2}$. After performing $Q_{t}^{2}$ and $Q_{t}^{\prime 2}$ integrations, $\mathscr{I}_{g g}$ takes the simplified form as follows:

$$
\begin{array}{r}
\mathscr{I}_{g g}=\frac{1}{\left(Y_{1}+Y_{2}\right)^{2}} \int \frac{d t_{1}}{t_{1}} \frac{d t_{2}}{t_{2}} \exp \left(-\frac{3 \alpha_{S}}{\pi} \Delta \eta\left|\ln \frac{t_{1}}{t_{2}}\right|\right) \\
\times T\left(\sqrt{\left|t_{1}\right|}, \mu\right) T\left(\sqrt{\left|t_{2}\right|}, \mu\right),
\end{array}
$$

where $\Delta \eta=\Delta \eta_{1}$ if $\left|t_{1}\right|>\left|t_{2}\right|$, but $\Delta \eta=\Delta \eta_{2}$ if $\left|t_{1}\right|<\left|t_{2}\right|$. In our computation of $\mathscr{L}_{g g}$, we have used this simplified form of $\mathscr{I}_{g g}$ taking $\Delta \eta_{1}=\Delta \eta_{2} \equiv \Delta \eta$.

\section{B. Feynman Rules and Graviton Propagator}

In this appendix, we give the Feynman rules for $V V G$ (where $V$ is a vector boson with mass $M_{V}$ ) and $f f G$ (where $f$ is a fermion with mass $M_{f}$ ) vertices [54]:
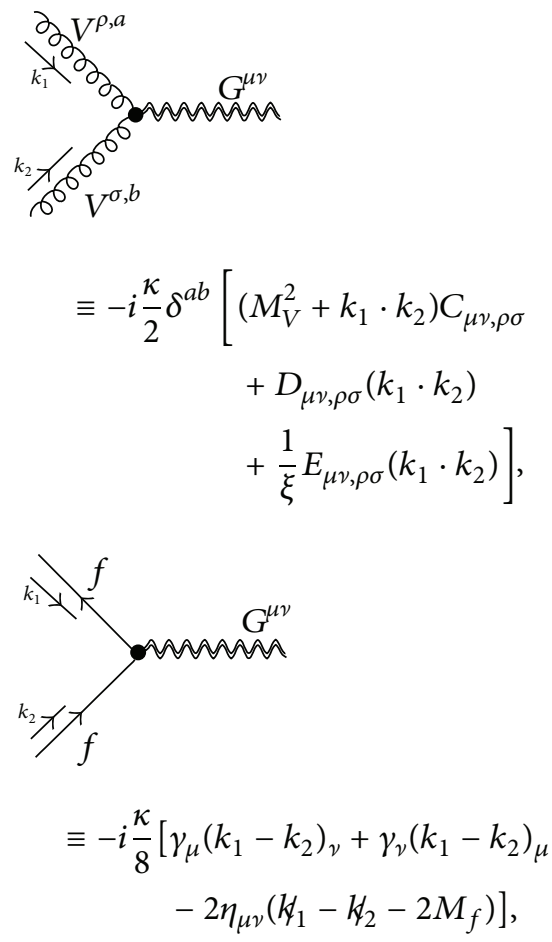

where $\kappa=\sqrt{16 \pi G_{N}}$. The $G_{N}=(4 \pi)^{n / 2} / R^{n} M_{S}^{2+n}$ is the $(4+n)$ dimensional Newton constant. All the tensors appear in the Feynman rules are defined as

$$
\begin{gathered}
C_{\mu \nu, \rho \sigma}=\eta_{\mu \rho} \eta_{\nu \sigma}+\eta_{\mu \sigma} \eta_{\nu \rho}-\eta_{\mu \nu} \eta_{\rho \sigma}, \\
\text { where } \eta_{\mu \nu}=\operatorname{diag}\{+1,-1,-1,-1\}, \\
D_{\mu \nu, \rho \sigma}\left(k_{1} \cdot k_{2}\right)=\eta_{\mu \nu} k_{1 \sigma} k_{2 \rho}-\left[\eta_{\mu \sigma} k_{1 \nu} k_{2 \rho}+\eta_{\mu \rho} k_{1 \sigma} k_{2 \nu}\right. \\
\left.-\eta_{\rho \sigma} k_{1 \mu} k_{2 \nu}+(\mu \longleftrightarrow \nu)\right], \\
E_{\mu \nu, \rho \sigma}\left(k_{1} \cdot k_{2}\right)=\eta_{\mu \nu}\left(k_{1 \rho} k_{1 \sigma}+k_{2 \rho} k_{2 \sigma}+k_{1 \rho} k_{2 \sigma}\right) \\
-\left[\eta_{\nu \sigma} k_{1 \mu} k_{1 \rho}+\eta_{\nu \rho} k_{2 \mu} k_{2 \sigma}+(\mu \longleftrightarrow \nu)\right] .
\end{gathered}
$$

The propagator for spin-2 KK graviton with mass $M_{G}$ and decay width $\Gamma_{G}$ is given by

$$
\Delta_{\mu \nu, \rho \sigma}(k)=\frac{(i / 2) B_{\mu \nu, \rho \sigma}(k)}{k^{2}-M_{G}^{2}+i \Gamma_{G} M_{G}},
$$


where

$$
\begin{aligned}
B_{\mu \nu, \rho \sigma}(k)= & \left(\eta_{\mu \rho}-\frac{k_{\mu} k_{\rho}}{M_{G}^{2}}\right)\left(\eta_{\nu \sigma}-\frac{k_{v} k_{\sigma}}{M_{G}^{2}}\right) \\
& +\left(\eta_{\mu \sigma}-\frac{k_{\mu} k_{\sigma}}{M_{G}^{2}}\right)\left(\eta_{\nu \rho}-\frac{k_{\nu} k_{\rho}}{M_{G}^{2}}\right) \\
& -\frac{2}{3}\left(\eta_{\mu \nu}-\frac{k_{\mu} k_{\nu}}{M_{G}^{2}}\right)\left(\eta_{\rho \sigma}-\frac{k_{\rho} k_{\sigma}}{M_{G}^{2}}\right) .
\end{aligned}
$$

Mass of the $k$ th $\mathrm{KK}$ mode is $M_{k}=4 \pi^{2} k^{2} / R^{2}$, and mass separation between two adjacent KK modes is a $\mathcal{O}(1 / R)$ term. Therefore, KK modes become quasicontinuous, and it is convenient to define $\mathrm{KK}$ state density. The number of $\mathrm{KK}$ states in the mass interval $M_{k}^{2}$ and $M_{k}^{2}+d M_{k}^{2}$ for $4+n$ dimensional space can be expressed as $\Delta k^{2}=\rho\left(M_{k}\right) d M_{k}^{2}$, where

$$
\rho\left(M_{k}\right)=\frac{R^{n} M_{k}^{n-2}}{(4 \pi)^{n / 2} \Gamma(n / 2)} .
$$

The effective interaction due to all KK states contributing to a physical process can be obtained after summing over all the propagators as follows:

$$
\mathscr{D}(\widehat{s})=\sum_{k} \frac{i}{\widehat{s}-M_{k}^{2}+i \varepsilon}=\int_{0}^{\infty} d M_{k}^{2} \rho\left(M_{k}\right) \frac{i}{\widehat{s}-M_{k}^{2}+i \varepsilon},
$$

where $\varepsilon=M_{G} \Gamma_{G}$ and $\widehat{s}=k^{2}$. The effective propagator $\mathscr{D}(\widehat{s})$ valid up to $M_{S}$ looks as

$$
\mathscr{D}(\widehat{s})=\frac{\widehat{s}^{n / 2-1} R^{n}}{\Gamma(n / 2)(4 \pi)^{n / 2}}\left[\pi+2 i I\left(\frac{M_{S}}{\sqrt{\widehat{s}}}\right)\right],
$$

where

$$
I\left(\frac{M_{S}}{\sqrt{\widehat{s}}}\right)=\left\{\begin{array}{cl}
-\sum_{k=1}^{n / 2-1} \frac{1}{2 k}\left(\frac{M_{S}}{\sqrt{\widehat{s}}}\right)^{2 k} & \text { for } n=\text { even } \\
-\frac{1}{2} \ln \left(\frac{M_{S}^{2}}{\widehat{s}}-1\right) & \\
-\sum_{k=1}^{(n-1) / 2} \frac{1}{2 k-1}\left(\frac{M_{S}}{\sqrt{\widehat{s}}}\right)^{2 k-1} & \\
+\frac{1}{2} \ln \left(\frac{M_{S}+\sqrt{\widehat{s}}}{M_{S}-\sqrt{\widehat{s}}}\right) & \text { for } n=\text { odd }
\end{array}\right.
$$

In the limit $M_{S}^{2} \gg s$, the effective propagator $\mathscr{D}(\widehat{s})$ becomes

$$
|\mathscr{D}(\widehat{s})|=\frac{16 \pi}{\mathcal{K}^{2} M_{S}^{4}} \mathscr{K} \quad \text { where } \mathscr{K}= \begin{cases}\ln \left(\frac{M_{S}^{2}}{\widehat{s}}\right) & \text { for } n=2 \\ \frac{2}{n-2} & \text { for } n>2 .\end{cases}
$$

In our analysis, we have used the exact form of $\mathscr{D}(\widehat{s})$ given in (B.7).

\section{Dilepton Background}

We consider the inelastic photoproduction of a lepton pair at the LHC shown in Figure 15 as follows:

$$
p p \longrightarrow X+\gamma \gamma+Y \longrightarrow X+l^{+} l^{-}+Y .
$$

A photon with momentum fraction $z_{1}\left(z_{2}\right)$ with respect to the quark momentum is emitted from the quark $q_{1}\left(q_{2}\right)$ with momentum fraction $x_{1}\left(x_{2}\right)$ of a proton. The total inelastic $p p \rightarrow l^{+} l^{-}$cross-section can be computed (this has been thoroughly computed in [59]) using the Weizsäcker-Williams equivalent photon approximation $[62,63]$ as given by

$$
\begin{aligned}
\sigma(S)=\sum_{q_{1}, q_{2}} & \int_{4 m_{l}^{2} / S}^{1} d x_{1} \int_{4 m_{l}^{2} / S x_{1}}^{1} d x_{2} \int_{4 m_{l}^{2} / S x_{1} x_{2}}^{1} d z_{1} \\
& \times \int_{4 m_{l}^{2} / S x_{1} x_{2} z_{1}}^{1} d z_{2} e_{q_{1}}^{2} e_{q_{2}}^{2} \\
& \times f_{q_{1} / p}\left(x_{1}, Q^{2}\right) f_{q_{2} / p}\left(x_{2}, Q^{2}\right) \\
& \times f_{\gamma / q_{1}}\left(z_{1}\right) f_{\gamma / q_{2}}\left(z_{2}\right) \widehat{\sigma}(\widehat{s}),
\end{aligned}
$$

where $q_{1}, q_{2}$ are the quarks (we denote $q \equiv\left\{q_{1}, q_{2}\right\}$ ), $m_{l}$ is the mass of the lepton, $e_{q}$ is the EM charge of $q$ in the unit of an electron's charge, and $\widehat{\sigma}$ is the subprocess cross-section with CM energy $\sqrt{\widehat{s}}\left(\widehat{s}=x_{1} x_{2} z_{1} z_{2} S, S\right.$ is the LHC CM energy). The quark density inside the proton is denoted as $f_{q / p}$. The $f_{\gamma / q}$ is the photon spectrum inside a quark and is given by

$$
f_{\gamma / q}(z)=\frac{\alpha_{e m}}{2 \pi} \frac{\left[1+(1-z)^{2}\right]}{z} \operatorname{In}\left(\frac{Q_{1}^{2}}{Q_{2}^{2}}\right) .
$$

We choose the scale $Q_{1}=\sqrt{\hat{s}} / 2$ and $Q_{2}=1 \mathrm{GeV}$. The subprocess cross-section for $\gamma \gamma \rightarrow l l$ is given by

$$
\widehat{\sigma}=\frac{4 \pi \alpha_{e m}^{2}\left(M_{W}^{2}\right)}{\widehat{s}}\left[\frac{3-\beta^{4}}{2} \ln \left(\frac{1+\beta}{1-\beta}\right)-2 \beta+\beta^{3}\right],
$$

where $\beta=\sqrt{1-4 m_{l}^{2} / \widehat{s}}$. We have used $\alpha_{e m}=1 / 137$ and $\alpha_{e m}^{2}\left(M_{W}^{2}\right)=1 / 128$ in our analysis.

The differential cross-section of the subprocess in the high energy limit is

$$
\frac{d \widehat{\sigma}}{d|\cos \theta|}=\frac{2 \pi \alpha_{e m}^{2}\left(M_{W}^{2}\right)}{\widehat{s}}\left(\frac{1+\cos ^{2} \theta}{\sin ^{2} \theta}\right),
$$

where $\theta$ is scattering angle of the lepton in the CM frame. We see that $d \widehat{\sigma} / d|\cos \theta| \rightarrow \infty$ if $|\cos \theta| \rightarrow 1$.

\section{Diphoton Background}

We compute the dominant background cross-section for the diphoton channe $g g \rightarrow \gamma \gamma$ via a quark loop using the $\gamma \gamma \rightarrow \gamma \gamma$ helicity amplitudes given in [60]. The differential cross-section of $g g \rightarrow \gamma \gamma$ background in the high energy 


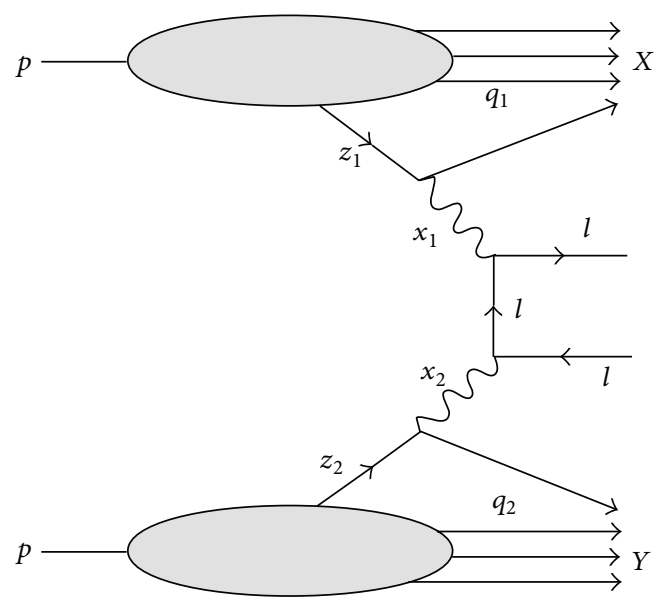

FIgURE 15: Inelastic photoproduction of a lepton pair at the LHC. The photons are coming from the quarks inside protons.

limit and for small $\theta$ (scattering angle of $\gamma$ in the CM frame) is given by

$$
\begin{aligned}
\frac{d \sigma}{d|\cos \theta|}= & \frac{1}{2} \cdot\left(\frac{1}{32 \pi \hat{s}}\right) \cdot\left(\frac{1}{8^{2}}\right) \cdot\left(\frac{1}{2^{2}}\right) \\
& \cdot 8 \cdot N_{f} N_{c} \cdot \alpha_{S}^{2} \alpha_{e m}^{2}|M|^{2} .
\end{aligned}
$$

Origin of some factors in the previous equation is once explained in Section 4.2, and we are not explaining them again. The factor $N_{f} N_{c}$ is the number of possible quarks that can contribute in the loop. The $|\mathscr{M}|^{2}$ is the sum of two helicity amplitudes, which are the only two surviving amplitudes in the high energy limit,

$$
|\mathscr{M}|^{2}=\left|\mathscr{M}_{++++}\right|^{2}+\left|\mathscr{M}_{+-+-}\right|^{2}=32 \operatorname{In}^{4}\left(\frac{2}{1+|\cos \theta|}\right) .
$$

\section{Acknowledgment}

The authors are grateful to Subhadip Mitra for many fruitful discussions and assistance in certain numerical aspects of this work.

\section{References}

[1] N. Arkani-Hamed, S. Dimopoulos, and G. R. Dvali, "The hierarchy problem and new dimensions at a millimeter," Physics Letters B, vol. 429, no. 3-4, p. 263, 1998.

[2] L. Randall and R. Sundrum, "Large mass hierarchy from a small extra dimension," Physical Review Letters, vol. 83, no. 17, pp. 3370-3373, 1999.

[3] C. Royon and RP220 Collaboration, "Project to install roman pot detectors at $220 \mathrm{~m}$ in ATLAS," http://arxiv.org/abs/ 0706.1796.

[4] M. G. Albrow, R. B. Appleby, and M. Arneodo, "The FP420 R\&D project: Higgs and new physics with forward protons at the LHC," Journal of Instrumentation, vol. 4, Article ID T10001, 2009.
[5] L. A. Harland-Lang, V. A. Khoze, M. G. Ryskin, and W. J. Stirling, "The phenomenology of central exclusive production at hadron colliders," European Physical Journal C, vol. 72, article 2110, 2012.

[6] M. G. Albrow, T. D. Coughlin, and J. R. Forshaw, "Central exclusive particle production at high energy hadron colliders," Progress in Particle and Nuclear Physics, vol. 65, no. 2, pp. 149184, 2010.

[7] A. D. Martin, M. G. Ryskin, and V. A. Khoze, "Forward physics at the LHC," Acta Physica Polonica B, vol. 40, pp. 1841-1876, 2009.

[8] R. A. Ryutin, "Exclusive double diffractive events: generalframework and prospects," European Physical Journal C, vol. 73, article 2443, 2013.

[9] Y. L. Dokshitzer, V. A. Khoze, and T. Sjostrand, "Rapidity gaps in Higgs production," Physics Letters B, vol. 274, no. 1, pp. 116-121, 1992.

[10] R. S. Fletcher and T. Stelzer, "Rapidity gap signals in Higgsboson production at the SSC," Physical Review D, vol. 48, no. 11, pp. 5162-5167, 1993.

[11] H. J. Lu and J. Milana, "Exclusive production of Higgs bosons in hadron colliders," Physical Review D, vol. 51, no. 11, pp. 61076113, 1995.

[12] T. L. Lungov and C. O. Escobar, "Using rapidity gaps to distinguish between Higgs boson production by W and gluon fusion," Physical Review D, vol. 53, no. 9, pp. 4857-4865, 1996.

[13] J. R. Cudell and O. F. Hernandez, "Particle production in a hadron collider rapidity gap: the Higgs case," Nuclear Physics B, vol. 471, no. 3, pp. 471-499, 1996.

[14] V. A. Khoze, A. D. Martin, and M. G. Ryskin, "The rapidity gap Higgs signal at LHC," Physics Letters B, vol. 401, no. 3-4, pp. 330336, 1997.

[15] V. A. Khoze, A. D. Martin, and M. G. Ryskin, "Can the Higgs be seen in rapidity gap events at the Tevatron or the LHC?" European Physical Journal C, vol. 14, no. 3, pp. 525-534, 2000.

[16] B. Cox, J. R. Forshaw, and B. Heinemann, "Double diffractive higgs and di-photon production at the Tevatron and LHC," Physics Letters B, vol. 540, no. 3-4, pp. 263-268, 2002.

[17] V. A. Petrov and R. A. Ryutin, "Exclusive double diffractive Higgs boson production at LHC," European Physical Journal C, vol. 36, no. 4, pp. 509-513, 2004.

[18] J. R. Forshaw, "Diffractive Higgs production: theory," http:// arxiv.org/abs/hep-ph/0508274.

[19] R. Maciula, R. Pasechnik, and A. Szczurek, "Exclusive $b \bar{b}$ pair production and irreducible background to the exclusive Higgs boson production," Physical Review D, vol. 82, no. 11, Article ID 114011, 7 pages, 2010.

[20] M. G. Albrow and A. Rostovtsev, "Searching for the Higgs boson at hadron colliders using the missing mass method," http://arxiv.org/abs/hep-ph/0009336.

[21] V. A. Khoze, A. D. Martin, and M. G. Ryskin, "Doublediffractive processes in high-resolution missing-mass experiments at the Tevatron," European Physical Journal C, vol. 19, no. 3, pp. 477-483, 2001, Erratum in European Physical Journal C, vol. 20, no. 3, p.599, 2001.

[22] A. B. Kaidalov, V. A. Khoze, A. D. Martin, and M. G. Ryskin, "Central exclusive diffractive production as a spin-parity analyser: from hadrons to Higgs," European Physical Journal C, vol. 31, no. 3, pp. 387-396, 2003.

[23] V. A. Khoze, A. D. Martin, and M. G. Ryskin, "Physics with tagged forward protons at the LHC," European Physical Journal C, vol. 24, no. 4, pp. 581-587, 2002. 
[24] B. E. Cox, "A review of forward proton tagging at $420 \mathrm{~m}$ at the LHC, and relevant results from the Tevatron and HERA," AIP Conference Proceedings, vol. 753, pp. 103-111, 2005.

[25] P. Lebiedowicz, R. Pasechnik, and A. Szczurek, "Measurement of exclusive production of scalar $X^{c 0}$ meson in proton(anti)proton collisions via $X^{c 0 \rightarrow \pi^{+} \pi^{-}}$decay," Physics Letters $B$, vol. 701, no. 4, pp. 434-444, 2011.

[26] L. A. Harland-Lang, V. A. Khoze, M. G. Ryskin, and W. J. Stirling, "Central exclusive meson pair production in the perturbative regime at hadron colliders," European Physical Journal C, vol. 71, article 1714, 2011.

[27] T. Aaltonen, A. Abulencia, J. Adelman et al., "Search for exclusive $\gamma \gamma$ production in Hadron-Hadron collisions," Physical Review Letters, vol. 99, no. 24, Article ID 242002, 7 pages, 2007.

[28] A. Abulencia, J. Adelman, T. Affolder et al., "Observation of exclusive electron-positron production in Hadron-Hadron collisions," vol. 98, no. 11, Article ID 112001, 7 pages, 2007.

[29] T. Aaltonen, J. Adelman, T. Akimoto et al., "Observation of exclusive charmonium production and $\gamma \gamma \rightarrow \mu^{+} \mu^{-}$in $p \bar{p}$ collisions at $\sqrt{s}=1.96 \mathrm{TeV}$,' Physical Review Letters, vol. 102, no. 24, Article ID 242001, 7 pages, 2009.

[30] T. Aaltonen, J. Adelman, T. Akimoto et al., "Observation of exclusive dijet production at the Fermilab Tevatron $\bar{p} p$ collider," Physical Review D, vol. 77, no. 5, Article ID 052004, 28 pages, 2008.

[31] V. M. Abazov, B. Abbott, M. Abolins et al., "High mass exclusive diffractive dijet production in $p \bar{p}$ collisions at $\sqrt{s}=1.96 \mathrm{TeV}$," Physics Letters B, vol. 705, no. 3, pp. 193-199, 2011.

[32] V. A. Khoze, A. D. Martin, and M. G. Ryskin, "Prospects for new physics observations in diffractive processes at the LHC and Tevatron," European Physical Journal C, vol. 23, no. 2, pp. 311-327, 2002.

[33] S. Heinemeyer, V. A. Khoze, M. G. Ryskin, W. J. Stirling, M. Tasevsky, and G. Weiglein, "Studying the MSSM Higgs sector by forward proton tagging at the LHC," European Physical Journal C, vol. 53, no. 2, pp. 231-256, 2008.

[34] R. Enberg and R. Pasechnik, "Associated central exclusive production of charged Higgs bosons," Physical Review D, vol. 83, no. 9, Article ID 095020, 9 pages, 2011.

[35] M. Chaichian, P. Hoyer, K. Huitu, V. A. Khoze, and A. D. Pilkington, "Searching for the triplet Higgs sector via central exclusive production at the LHC," Journal of High Energy Physics, vol. 2009, no. 5, article 11, 2009.

[36] V. P. Goncalves and W. K. Sauter, "Radion production in exclusive processes at CERN LHC," Physical Review D, vol. 82, no. 5, Article ID 056009, 6 pages, 2010.

[37] A. V. Kisselev, V. A. Petrov, and R. A. Ryutin, "Production of radions and massive gravitons at LHC," Conference: C05-06-22, pp. $49-58$.

[38] P. J. Bussey, T. D. Coughlin, J. R. Forshaw, and A. D. Pilkington, "Central exclusive production of longlived gluinos at the LHC," Journal of High Energy Physics, vol. 2006, no. 11, article 27, 2006.

[39] A. V. Kisselev, V. A. Petrov, and R. A. Ryutin, "5-dimensional quantum gravity effects in exclusive double diffractive events," Physics Letters B, vol. 630, no. 3-4, pp. 100-107, 2005.

[40] G. Aad, T. Abajyan, B. Abbott et al., "Search for contact interactions and large extra dimensions in dilepton events from pp collisions at $\sqrt{s}=7 \mathrm{TeV}$ with the ATLAS detector," Physical Review D, vol. 87, no. 1, Article ID 015010, 25 pages, 2013.

[41] G. Aad, T. Abajyan, B. Abbott et al., "Search for extra dimensions in diphoton events from proton-proton collisions at $\sqrt{s}=7 \mathrm{TeV}$ in the ATLAS detector at the LHC," New Journal of Physics, vol. 15, Article ID 043007, 2013.

[42] S. Chatrchyan, V. Khachatryan, A. M. Sirunyan et al., "Search for large extra dimensions in dimuon and dielectron events in pp collisions at $\sqrt{s}=7 \mathrm{TeV}$,' Physics Letters B, vol. 711, no. 1, pp. 15-34, 2012.

[43] S. Chatrchyan, V. Khachatryan, A. M. Sirunyan et al., "Search for signatures of extra dimensions in the diphoton mass spectrum at the large Hadron vollider," Physical Review Letters, vol. 108, no. 11, Article ID 111801, 16 pages, 2012.

[44] A. G. Shuvaev, K. J. Golec-Biernat, A. D. Martin, and M. G. Ryskin, "Off-diagonal distributions fixed by diagonal partons at small $x$ and $\zeta$," Physical Review D, vol. 60, no. 1, Article ID 014015, 6 pages, 1999.

[45] J. D. Bjorken, "Rapidity gaps and jets as a new-physics signature in very-high-energy hadron-hadron collisions," Physical Review D, vol. 47, no. 1, pp. 101-113, 1993.

[46] E. Gotsman, E. M. Levin, and U. Maor, "Large rapidity gaps in pp collisions," Physics Letters B, vol. 309, no. 1-2, pp. 199-204, 1993.

[47] V. A. Khoze, A. D. Martin, and M. G. Ryskin, "Soft diffraction and the elastic slope at Tevatron and LHC energies: a multiPomeron approach," European Physical Journal C, vol. 18, no. 1, pp. 167-179, 2000.

[48] A. B. Kaidalov, V. A. Khoze, A. D. Martin, and M. G. Ryskin, "Probabilities of rapidity gaps in high energy interactions," European Physical Journal C, vol. 21, no. 3, pp. 521-529, 2001.

[49] V. A. Khoze, A. D. Martin, and M. G. Ryskin, "On the role of hard rescattering in exclusive diffractive Higgs production," Journal of High Energy Physics, vol. 2006, no. 5, article 36, 2006.

[50] M. G. Ryskin, A. D. Martin, and V. A. Khoze, "Soft diffraction at the LHC: a partonic interpretation," European Physical Journal C, vol. 54, no. 2, pp. 199-217, 2008.

[51] E. Gotsman, E. Levin, U. Maor, and J. S. Miller, "A QCD motivated model for soft interactions at high energies," European Physical Journal C, vol. 57, no. 4, pp. 689-709, 2008.

[52] M. G. Ryskin, A. D. Martin, and V. A. Khoze, "Soft processes at the LHC, II: soft-hard factorisation breaking and gap survival," European Physical Journal C, vol. 60, no. 2, pp. 265-272, 2009.

[53] A. D. Martin, W. J. Stirling, R. S. Thorne, and G. Watt, "Uncertainties on $\alpha \mathrm{S}$ in global PDF analyses and implications for predicted hadronic cross sections," European Physical Journal C, vol. 64, no. 4, pp. 653-680, 2009.

[54] T. Han, J. D. Lykken, and R. J. Zhang, "Kaluza-Klein states from large extra dimensions," Physical Review D, vol. 59, no. 10, Article ID 105006, 14 pages, 1999.

[55] L. D. Landau, "The moment of a 2-photon system," Doklady Akademii Nauk SSSR, vol. 60, p. 207, 1948.

[56] C. N. Yang, "Selection rules for the dematerialization of a particle into two photons," Physical Review, vol. 77, no. 2, pp. $242-245,1950$

[57] L. A. Harland-Lang, V. A. Khoze, M. G. Ryskin, and W. J. Stirling, "Standard candle central exclusive processes at the Tevatron and LHC," European Physical Journal C, vol. 69, no. 1-2, pp. 179-199, 2010.

[58] V. A. Khoze, A. D. Martin, M. G. Ryskin, and W. J. Stirling, "Diffractive $\gamma \gamma$ production at hadron colliders," European Physical Journal C, vol. 38, no. 4, pp. 475-482, 2005.

[59] M. Drees, R. M. Godbole, M. Nowakowski, and S. D. Rindani, " $\gamma \gamma$ processes at high energy $p p$ collider," Physical Review D, vol. 50, no. 3, pp. 2335-2338, 1994. 
[60] G. Jikia and A. Tkabladze, "Photon-photon scattering at the photon linear collider," Physics Letters B, vol. 323, no. 3-4, pp. 453-458, 1994.

[61] J. R. Forshaw and M. G. Ryskin, "Diffractive vector meson production at large momentum transfer," Zeitschrift für Physik C, vol. 68, no. 1, pp. 137-147, 1995.

[62] C. F. von Weizsacker, "Ausstrahlung bei Stößen sehr schneller Elektronen," Zeitschrift für Physik, vol. 88, no. 9-10, pp. 612-625, 1934.

[63] E. J. Williams, "Nature of the high energy particles of penetrating radiation and status of ionization and radiation formulae," Physical Review, vol. 45, no. 10, pp. 729-730, 1934. 

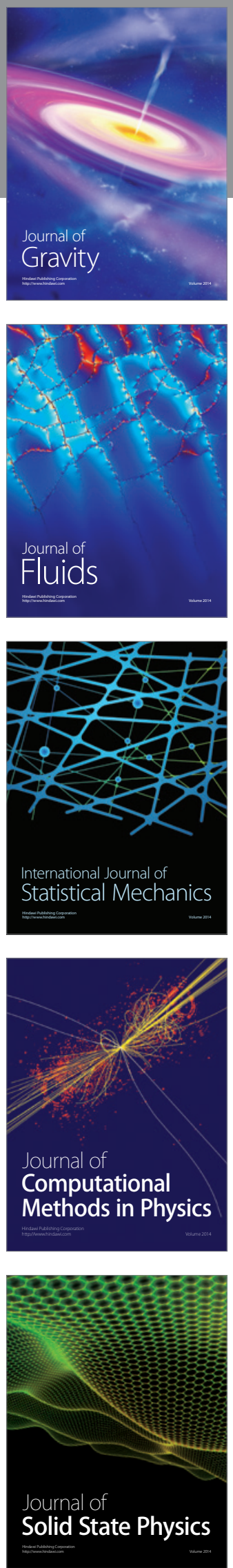

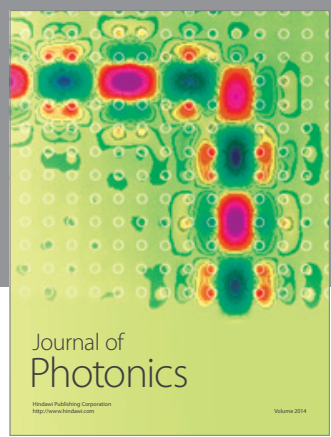

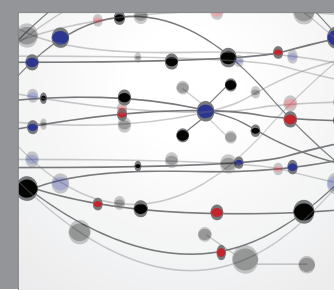

The Scientific World Journal

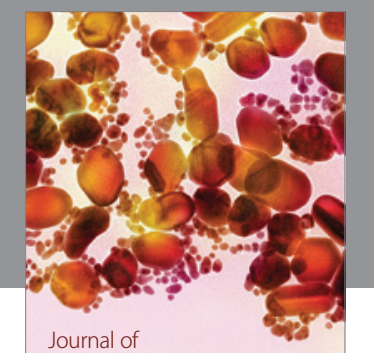

Soft Matter
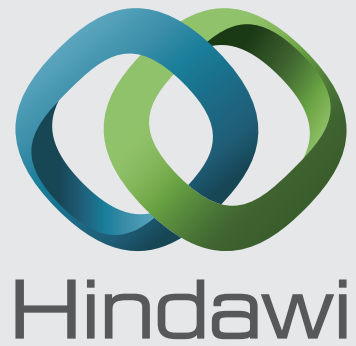

Submit your manuscripts at

http://www.hindawi.com
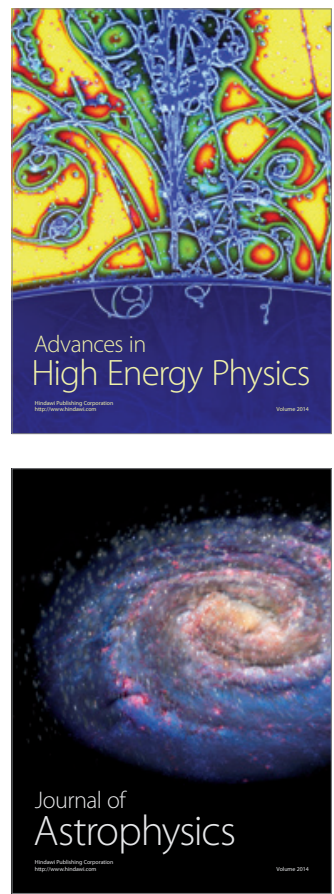
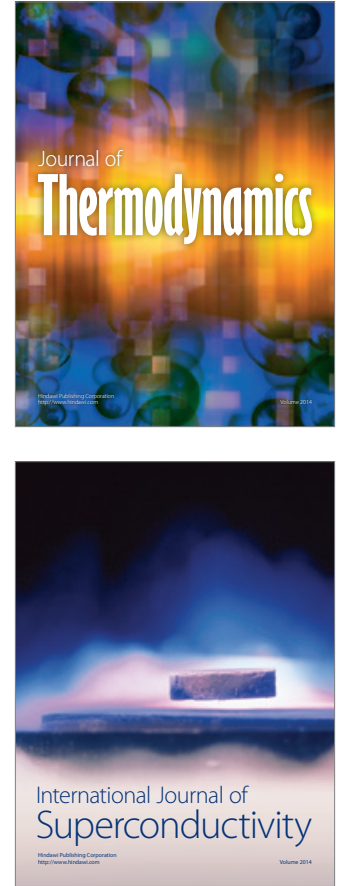
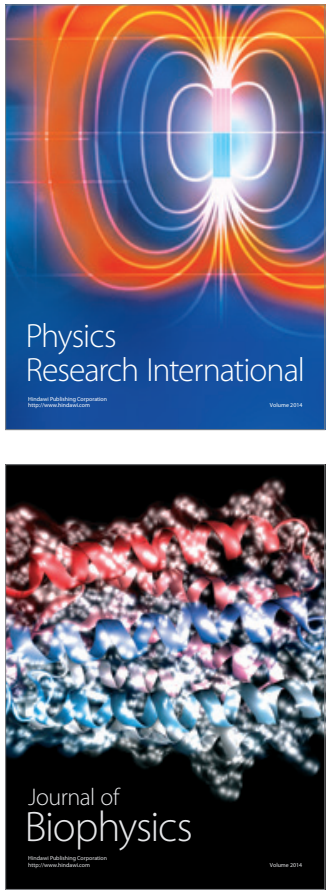
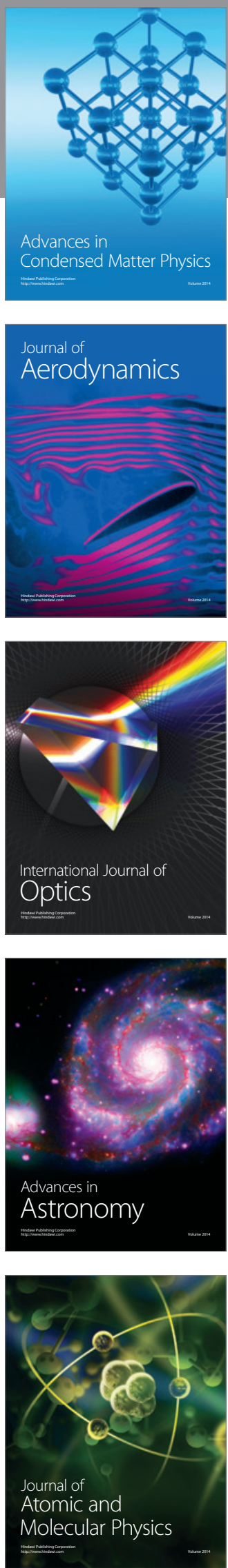